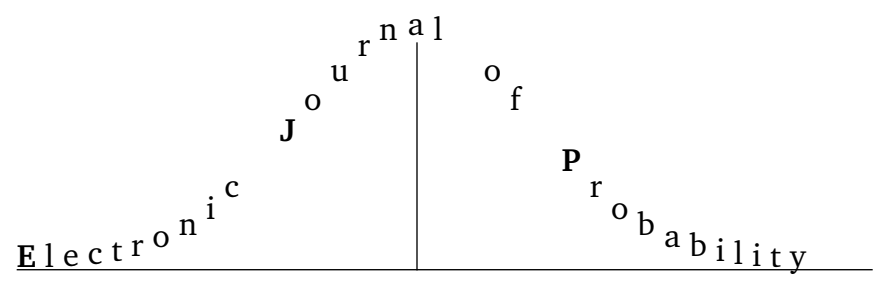

Vol. 16 (2011), Paper no. 11, pages 314-346.

Journal URL

http://www.math.washington.edu/ ejpecp/

\title{
Bulk scaling limit of the Laguerre ensemble
}

\author{
Stéphanie Jacquot* $\quad$ Benedek Valkó
}

\begin{abstract}
We consider the $\beta$-Laguerre ensemble, a family of distributions generalizing the joint eigenvalue distribution of the Wishart random matrices. We show that the bulk scaling limit of these ensembles exists for all $\beta>0$ for a general family of parameters and it is the same as the bulk scaling limit of the corresponding $\beta$-Hermite ensemble.
\end{abstract}

Key words: Random matrices, eigenvalues, Laguerre ensemble, Wishart ensemble, bulk scaling limit.

AMS 2000 Subject Classification: Primary 60B20; Secondary: 60G55, 60H10.

Submitted to EJP on June 7, 2010, final version accepted January 2, 2011.

\footnotetext{
*University of Cambridge, Statistical Laboratory, Centre for Mathematical Sciences, Wilberforce Road, Cambridge, CB3 OWB, UK. S.M.Jacquot@statslab.cam.ac.uk

†Department of Mathematics, University of Wisconsin - Madison, WI 53705, USA. valko@math.wisc.edu B.Valkó was partially supported by the NSF Grant DMS-09-05820.
} 


\section{Introduction}

The Wishart ensemble is one of the first studied random matrix models, introduced by Wishart in 1928 [15]. It describes the joint eigenvalue distribution of the $n \times n$ random symmetric matrix $M=A A^{*}$ where $A$ is an $n \times(m-1)$ matrix with i.i.d. standard normal entries. We can use real, complex or real quaternion standard normal random variables as ingredients. Since we are only interested in the eigenvalues, we can assume $m-1 \geq n$. Then the joint eigenvalue density on $\mathbb{R}_{+}^{n}$ exists and it is given by the following formula for all three versions:

$$
\frac{1}{Z_{n, m+1}^{\beta}} \prod_{j<k}\left|\lambda_{j}-\lambda_{k}\right|^{\beta} \prod_{k=1}^{n} \lambda_{k}^{\frac{\beta}{2}(m-n)-1} e^{-\frac{\beta}{2} \lambda_{k}} .
$$

Here $\beta=1,2$ and 4 correspond to the real, complex and quaternion cases respectively and $Z_{n, m+1}^{\beta}$ is an explicitly computable constant.

The density (1) defines a distribution on $\mathbb{R}_{+}^{n}$ for any $\beta>0, n \in \mathbb{N}$ and $m>n$ with a suitable $Z_{n, m+1}^{\beta}$. The resulting family of distributions is called the $\beta$-Laguerre ensemble. Note that we intentionally shifted the parameter $m$ by one as this will result in slightly cleaner expressions later on.

Another important family of distributions in random matrix theory is the $\beta$-Hermite (or Gaussian) ensemble. It is described by the density function

$$
\frac{1}{\tilde{Z}_{n}^{\beta}} \prod_{1 \leq j<k \leq n}\left|\lambda_{j}-\lambda_{k}\right|^{\beta} \prod_{k=1}^{n} e^{-\frac{\beta}{4} \lambda_{k}^{2}} .
$$

on $\mathbb{R}^{n}$. For $\beta=1,2$ and 4 this gives the joint eigenvalue density of the Gaussian orthogonal, unitary and symplectic ensembles. It is known that if we rescale the ensemble by $\sqrt{n}$ then the empirical spectral density converges to the Wigner semicircle distribution $\frac{1}{2 \pi} \sqrt{4-x^{2}} 1_{[-2,2]}(x)$. In [13] the authors derive the bulk scaling limit of the $\beta$-Hermite ensemble, i.e. the point process limit of the spectrum it is scaled around a sequence of points away from the edges.

Theorem 1 (Valkó and Virág [13]). If $\mu_{n}$ is a sequence of real numbers satisfying $n^{1 / 6}\left(2 \sqrt{n}-\left|\mu_{n}\right|\right) \rightarrow$ $\infty$ as $n \rightarrow \infty$ and $\Lambda_{n}^{H}$ is a sequence of random vectors with density (2) then

$$
\sqrt{4 n-\mu_{n}^{2}}\left(\Lambda_{n}^{H}-\mu_{n}\right) \Rightarrow \text { Sine }_{\beta}
$$

where Sine $_{\beta}$ is a discrete point process with density $(2 \pi)^{-1}$.

Note that the condition on $\mu_{n}$ means that we are in the bulk of the spectrum, not too close to the edge. The limiting point process Sine ${ }_{\beta}$ can be described as a functional of the Brownian motion in the hyperbolic plane or equivalently via a system of stochastic differential equations (see Subsection 2.3 for details).

The main result of the present paper provides the point process limit of the Laguerre ensemble in the bulk. In order to understand the order of the scaling parameters, we first recall the classical results about the limit of the empirical spectral measure for the Wishart matrices. If $m / n \rightarrow \gamma \in[1, \infty)$ then with probability one the scaled empirical spectral measures $v_{n}=\frac{1}{n} \sum_{k=1}^{n} \delta_{\lambda_{k} / n}$ converge weakly to the Marchenko-Pastur distribution which is a deterministic measure with density

$$
\tilde{\sigma}^{\gamma}(x)=\frac{\sqrt{\left(x-a^{2}\right)\left(b^{2}-x\right)}}{2 \pi x} 1_{\left[a^{2}, b^{2}\right]}(x), \quad a=a(\gamma)=\gamma^{1 / 2}-1, b=b(\gamma)=1+\gamma^{1 / 2} .
$$


This can be proved by the moment method or using Stieltjes-transform. (See [7] for the original proof and [5] for the general $\beta$ case).

Now we are ready to state our main theorem:

Theorem 2 (Bulk limit of the Laguerre ensemble). Fix $\beta>0$, assume that $m / n \rightarrow \gamma \in[1, \infty)$ and let $c \in\left(a^{2}, b^{2}\right)$ for $a=a(\gamma), b=b(\gamma)$ defined in (4). Let $\Lambda_{n}^{L}$ denote the point process given by (1). Then

$$
2 \pi \tilde{\sigma}^{\gamma}(c)\left(\Lambda_{n}^{L}-c n\right) \Rightarrow \text { Sine }_{\beta}
$$

where Sine $_{\beta}$ is the bulk scaling limit of the $\beta$-Hermite ensemble and $\tilde{\sigma}^{\gamma}$ is defined in (4).

We will actually prove a more general version of this theorem: we will also allow the cases when $m / n \rightarrow \infty$ or when the center of the scaling gets close to the spectral edge. See Theorem 9 in Subsection 2.2 for the details.

Although this statement has been known for the classical cases $(\beta=1,2$ and 4) [8], this is the first proof for general $\beta$. Our approach relies on the tridiagonal matrix representation of the Laguerre ensemble introduced by Dumitriu and Edelman [1] and the techniques introduced in [13].

There are various other ways one can generalize the classical Wishart ensembles. One possibility is that instead of normal distribution one uses more general real or complex distributions in the construction described at the beginning of this section. It has been conjectured that the bulk scaling limit for these generalized Wishart matrices would be the same as in the $\beta=1$ and 2 cases for the Laguerre ensemble. The recent papers of Tao and Vu [12] and Erdös et al. [3] prove this conjecture for a wide range of distributions (see [12] and [3] for the exact conditions).

Our theorem completes the picture about the point process scaling limits of the Laguerre ensemble. The scaling limit at the soft edge has been proved in [9], where the edge limit of the Hermite ensemble was also treated.

Theorem 3 (Ramírez, Rider and Virág [9]). If $m>n \rightarrow \infty$ then

$$
\frac{(m n)^{1 / 6}}{(\sqrt{m}+\sqrt{n})^{4 / 3}}\left(\Lambda_{n}^{L}-(\sqrt{n}+\sqrt{m})^{2}\right) \Rightarrow \operatorname{Airy}_{\beta}
$$

where Airy $_{\beta}$ is a discrete simple point process given by the eigenvalues of the stochastic Airy operator

$$
\mathscr{H}_{\beta}=-\frac{d^{2}}{d x^{2}}+x+\frac{2}{\sqrt{\beta}} b_{x}^{\prime} .
$$

Here $b_{x}^{\prime}$ is white noise and the eigenvalue problem is set up on the positive half line with initial conditions $f(0)=0, f^{\prime}(0)=1$. A similar limit holds at the lower edge: if $\lim \inf m / n>1$ then

$$
\frac{(m n)^{1 / 6}}{(\sqrt{m}-\sqrt{n})^{4 / 3}}\left((\sqrt{m}-\sqrt{n})^{2}-\Lambda_{n}^{L}\right) \Rightarrow \operatorname{Airy}_{\beta} .
$$

Remark 4. The lower edge result is not stated explicitly in [9], but it follows by a straightforward modification of the proof of the upper edge statement. Note that the condition $\lim \inf m / n>1$ is not optimal, the statement is expected to hold with $m-n \rightarrow \infty$. This has been known for the classical cases $\beta=1,2,4[8]$. 
If $m-n \rightarrow a \in(0, \infty)$ then the lower edge of the spectrum is pushed to 0 and it becomes a 'hard' edge. The scaling limit in this case was proved in [10].

Theorem 5 (Ramírez and Rider [10]). If $m-n \rightarrow a \in(0, \infty)$ then

$$
n \Lambda_{n}^{L} \Rightarrow \Theta_{\beta, a}
$$

where $\Theta_{\beta, a}$ is a simple point process that can be described as the sequence of eigenvalues of a certain random operator (the Bessel operator).

In the next section we discuss the tridiagonal representation of the Laguerre ensemble, recall how to count eigenvalues of a tridiagonal matrix and state a more general version of our theorem. Section 3 will contain the outline of the proof while the rest of the paper deals with the details of the proof.

\section{Preparatory steps}

\subsection{Tridiagonal representation}

In [1] Dumitriu and Edelman proved that the $\beta$-Laguerre ensemble can be represented as joint eigenvalue distributions for certain random tridiagonal matrices.

Lemma 6 (Dumitriu, Edelman [1]). Let $A_{n, m}$ be the following $n \times n$ bidiagonal matrix:

$$
A_{n, m}=\frac{1}{\sqrt{\beta}}\left[\begin{array}{ccccc}
\tilde{\chi}_{\beta(m-1)} & & & & \\
\chi_{\beta(n-1)} & \tilde{\chi}_{\beta(m-2)} & & & \\
& \ddots & \ddots & & \\
& & \chi_{\beta \cdot 2} & \tilde{\chi}_{\beta(m-n+1)} & \tilde{\chi}_{\beta(m-n)}
\end{array}\right] \text {. }
$$

where $\chi_{\beta a}, \tilde{\chi}_{\beta b}$ are independent chi-distributed random variables with the appropriate parameters $(1 \leq$ $a \leq n-1, m-1 \leq b \leq m-n)$. Then the eigenvalues of the tridiagonal matrix $A_{n, m} A_{n, m}^{T}$ are distributed according to the density (1).

If we want to find the bulk scaling limit of the eigenvalues of $A_{n, m} A_{n, m}^{T}$ then it is sufficient to understand the scaling limit of the singular values of $A_{n, m}$. The following simple lemma will be a useful tool for this.

Lemma 7. Suppose that $B$ is an $n \times n$ bidiagonal matrix with $a_{1}, a_{2}, \ldots, a_{n}$ in the diagonal and $b_{1}, b_{2}, \ldots, b_{n-1}$ below the diagonal. Consider the $2 n \times 2 n$ symmetric tridiagonal matrix $M$ which has zeros in the main diagonal and $a_{1}, b_{1}, a_{2}, b_{2}, \ldots, a_{n}$ in the off-diagonal. If the singular values of $B$ are $\lambda_{1}, \lambda_{2}, \ldots, \lambda_{n}$ then the eigenvalues of $M$ are $\pm \lambda_{i}, i=1 \ldots n$.

We learned about this trick from [2], we reproduce the simple proof for the sake of the reader.

Proof. Consider the matrix $\tilde{B}=\left[\begin{array}{cc}0 & B^{T} \\ B & 0\end{array}\right]$. If $B u=\lambda_{i} v$ and $B^{T} v=\lambda_{i} u$ then $[u, \pm v]^{T}$ is an eigenvector of $\tilde{B}$ with eigenvalue $\pm \lambda_{i}$. Let $C$ be the permutation matrix corresponding to $(2,4, \ldots, 2 n, 1,3, \ldots, 2 n-1)$. Then $C^{T} \tilde{B} C$ is exactly the tridiagonal matrix described in the lemma and its eigenvalues are exactly $\pm \lambda_{i}, i=1 \ldots n$. 
Because of the previous lemma it is enough to study the eigenvalues of the $(2 n) \times(2 n)$ tridiagonal matrix

$$
\tilde{A}_{n, m}=\frac{1}{\sqrt{\beta}}\left[\begin{array}{ccccccc}
0 & \tilde{\chi}_{\beta(m-1)} & & & & & \\
\tilde{\chi}_{\beta(m-1)} & 0 & \chi_{\beta(n-1)} & & & & \\
& \chi_{\beta(n-1)} & 0 & \tilde{\chi}_{\beta(m-2)} & & & \\
& & \ddots & \ddots & \ddots & & \\
& & & \tilde{\chi}_{\beta(m-n+1)} & 0 & \chi_{\beta} & \\
& & & & \chi_{\beta} & 0 & \tilde{\chi}_{\beta(m-n)}
\end{array}\right]
$$

The main advantage of this representation, as opposed to studying the tridiagonal matrix $A_{n, m} A_{n, m}^{T}$, is that here the entries are independent modulo symmetry.

Remark 8. Assume that $\left[u_{1}, v_{1}, u_{2}, v_{2}, \ldots, u_{n}, v_{n}\right]^{T}$ is an eigenvector for $\tilde{A}_{n, m}$ with eigenvalue $\lambda$. Then $\left[u_{1}, u_{2}, \ldots, u_{n}\right]^{T}$ is and eigenvector for $A_{n, m}^{T} A_{n, m}$ with eigenvalue $\lambda^{2}$ and $\left[v_{1}, v_{2}, \ldots, v_{n}\right]^{T}$ is an eigenvector for $A_{n, m} A_{n, m}^{T}$ with eigenvalue $\lambda^{2}$.

\subsection{Bulk limit of the singular values}

We can compute the asymptotic spectral density of $\tilde{A}_{n, m}$ from the Marchenko-Pastur distribution. If $m / n \rightarrow \gamma \in[1, \infty$ ) then the asymptotic density (when scaled with $\sqrt{n}$ ) is

$$
\begin{aligned}
\sigma^{\gamma}(x) & =2|x| \tilde{\sigma}^{\gamma}\left(x^{2}\right)=\frac{\sqrt{\left(x^{2}-a^{2}\right)\left(b^{2}-x^{2}\right)}}{\pi|x|} 1_{[a, b]}(|x|) \\
& =\frac{\sqrt{(x-a)(x+a)(b-x)(b+x)}}{\pi|x|} 1_{[a, b]}(|x|) .
\end{aligned}
$$

This means that the spectrum of $\tilde{A}_{n, m}$ in $\mathbb{R}^{+}$is asymptotically concentrated on the interval $[\sqrt{m}-$ $\sqrt{n}, \sqrt{m}+\sqrt{n}]$. We will scale around $\mu_{n} \in(\sqrt{m}-\sqrt{n}, \sqrt{m}+\sqrt{n})$ where $\mu_{n}$ is chosen in a way that it is not too close to the edges. Near $\mu_{n}$ the asymptotic eigenvalue density should be close to $\sigma^{m / n}\left(\mu_{n} / \sqrt{n}\right)$ which explains the choice of the scaling parameters in the following theorem.

Theorem 9. Fix $\beta>0$ and suppose that $m=m(n)>n$. Let $\Lambda_{n}$ denote the set of eigenvalues of $\tilde{A}_{n, m}$ and set

$$
n_{0}=\frac{\pi^{2}}{4} n \sigma^{m / n}\left(\mu_{n} n^{-1 / 2}\right)^{2}-\frac{1}{2}, \quad n_{1}=n-\frac{\pi^{2}}{4} n \sigma^{m / n}\left(\mu_{n} n^{-1 / 2}\right)^{2}
$$

Assume that as $n \rightarrow \infty$ we have

$$
n_{1}^{1 / 3} n_{0}^{-1} \rightarrow 0
$$

and

$$
\liminf _{n \rightarrow \infty} m / n>1 \quad \text { or } \quad \lim _{n \rightarrow \infty} m / n=1 \text { and } \liminf _{n \rightarrow \infty} \mu_{n} / \sqrt{n}>0 .
$$

Then

$$
4 \sqrt{n_{0}}\left(\Lambda_{n}-\mu_{n}\right) \Rightarrow \text { Sine }_{\beta} .
$$


The extra $1 / 2$ in the definition of $n_{0}$ is introduced to make some of the forthcoming formulas nicer. We also note that the following identities hold:

$$
n_{0}+\frac{1}{2}=\frac{2(m+n) \mu_{n}^{2}-(m-n)^{2}-\mu_{n}^{4}}{4 \mu_{n}^{2}}, \quad n_{1}=\frac{\left(m-n-\mu_{n}^{2}\right)^{2}}{4 \mu_{n}^{2}} .
$$

Note that we did not assume that $m / n$ converges to a constant or that $\mu_{n}=\sqrt{c} \sqrt{n}$. By the discussions at the beginning of this section $\left(\Lambda_{n} \cap \mathbb{R}^{+}\right)^{2}$ is distributed according to the Laguerre ensemble. If we assume that $m / n \rightarrow \gamma$ and $\mu_{n}=\sqrt{c} \sqrt{n}$ with $c \in\left(a(\gamma)^{2}, b(\gamma)^{2}\right)$ then both (9) and (10) are satisfied. Since in this case $n_{0} n^{-1} \rightarrow \tilde{\sigma}^{\gamma}(c)$ the result of Theorem 9 implies Theorem 2 .

Remark 10. We want prove that the weak limit of $4 \sqrt{n_{0}}\left(\Lambda_{n}-\mu_{n}\right)$ is Sine ${ }_{\beta}$, thus it is sufficient to prove that for any subsequence of $n$ there is a further subsequence so that the limit in distribution holds. Because of this by taking an appropriate subsequence we may assume that

$$
m / n \rightarrow \gamma \in[1, \infty], \quad \text { and if } m / n \rightarrow 1 \text { then } \quad \mu_{n} / \sqrt{n} \rightarrow c \in(0,2] .
$$

These assumptions imply that for $m_{1}=m-n+n_{1}$ we have

$$
\liminf _{n \rightarrow \infty} m_{1} / n>0 \text {. }
$$

One only needs to check this in the $m / n \rightarrow 1$ case, when from (13) and the definition of $n_{1}$ we get $n_{1} / n \rightarrow c>0$.

Remark 11. The conditions of Theorem 9 are optimal if $\lim \inf m / n>1$ and the theorem provides a complete description of the possible point process scaling limits of $\Lambda_{n}^{L}$. To see this first note that using $\Lambda_{n}^{L}=\left(\Lambda_{n} \cap \mathbb{R}^{+}\right)^{2}$ we can translate the edge scaling limit of Theorem 3 to get

$$
\frac{2(m n)^{1 / 6}}{(\sqrt{m} \pm \sqrt{n})^{1 / 3}}\left(\Lambda_{n}-(\sqrt{m} \pm \sqrt{n})\right) \Rightarrow \pm \operatorname{Airy}_{\beta} .
$$

If $\liminf m / n>1$ then by the previous remark we may assume $\lim m / n=\gamma \in(1, \infty]$. Then the previous statement can be transformed into $n^{1 / 6}\left(\Lambda_{n}-(\sqrt{m} \pm \sqrt{n})\right) \stackrel{d}{\Longrightarrow} \Xi$ where $\Xi$ is a a linear transformation of Airy ${ }_{\beta}$. From this it is easy to check that if $n_{1}^{1 / 3} n_{0}^{-1} \rightarrow c \in(0, \infty]$ then we need to scale $\Lambda_{n}-\mu_{n}$ with $n^{1 / 6}$ to get a meaningful limit (and the limit is a linear transformation of Airy ${ }_{\beta}$ ) and if $n_{1}^{1 / 3} n_{0}^{-1} \rightarrow 0$ then we get the bulk case.

If $m / n \rightarrow 1$ then the condition (10) is suboptimal, this is partly due to the fact that the lower soft edge limit in this case is not available. Assuming $\liminf m-n>0$ the statement should be true with the following condition instead of (10):

$$
\mu_{n} \sqrt{n}(m-n)^{-1 / 3}-\frac{1}{2}(m-n)^{2 / 3} \rightarrow \infty
$$

It is easy to check that this condition is necessary for the bulk scaling limit. By choosing an appropriate subsequence we may assume that $m-n \rightarrow a>0$ or $m-n \rightarrow \infty$. Then if (16) does not hold then we can use Theorem 5 (if $m-n \rightarrow a>0$ ) or (15) (if $m-n \rightarrow \infty$ ) to show that an appropriately scaled version of $\Lambda_{n}-\mu_{n}$ converges to a shifted copy of the hard edge or soft edge limiting point process and thus it cannot converge to $\operatorname{Sine}_{\beta}$. 


\subsection{The Sine ${ }_{\beta}$ process}

The distribution of the point process Sine $_{\beta}$ from Theorem 1 was described in [13] as a functional of the Brownian motion in the hyperbolic plane (the Brownian carousel) or equivalently via a system of stochastic differential equations. We review the latter description here. Let $Z$ be a complex Brownian motion with i.i.d. standard real and imaginary parts. Consider the strong solution of the following one parameter system of stochastic differential equations for $t \in[0,1), \lambda \in \mathbb{R}$ :

$$
d \alpha_{\lambda}=\frac{\lambda}{2 \sqrt{1-t}} d t+\frac{\sqrt{2}}{\sqrt{\beta(1-t)}} \Re\left[\left(e^{-i \alpha_{\lambda}}-1\right) d Z\right], \quad \alpha_{\lambda}(0)=0 .
$$

It was proved in [13] that for any given $\lambda$ the limit $N(\lambda)=\frac{1}{2 \pi} \lim _{t \rightarrow 1} \alpha_{\lambda}(t)$ exists, it is integer valued a.s. and $N(\lambda)$ has the same distribution as the counting function of the point process Sine $\beta$ evaluated at $\lambda$. Moreover, this is true for the joint distribution of $\left(N\left(\lambda_{i}\right), i=1, \ldots, d\right)$ for any fixed vector $\left(\lambda_{i}, i=1, \ldots, d\right)$. Recall that the counting function at $\lambda>0$ gives the number of points in the interval $(0, \lambda]$, and negative the number of points in $(\lambda, 0]$ for $\lambda<0$.

\subsection{Counting eigenvalues of tridiagonal matrices}

Assume that the tridiagonal $k \times k$ matrix $M$ has positive off-diagonal entries.

$$
M=\left[\begin{array}{ccccc}
a_{1} & b_{1} & & & \\
c_{1} & a_{2} & b_{2} & & \\
& \ddots & \ddots & & \\
& & c_{k-2} & a_{k-1} & b_{k-1} \\
& & & c_{k-1} & a_{k}
\end{array}\right], \quad b_{i}>0, c_{i}>0 .
$$

If $u=\left[u_{1}, \ldots, u_{k}\right]^{T}$ is an eigenvector corresponding to $\lambda$ then we have

$$
c_{\ell-1} u_{\ell-1}+a_{\ell} u_{\ell}+b_{\ell} u_{\ell+1}=\lambda u_{\ell}, \quad \ell=1, \ldots k
$$

where we can we set $u_{0}=u_{k+1}=0$ (with $c_{0}, b_{k}$ defined arbitrarily). This gives a single term recursion on $\mathbb{R} \cup\{\infty\}$ for the ratios $r_{\ell}=\frac{u_{\ell+1}}{u_{\ell}}$ :

$$
r_{0}=\infty, \quad r_{\ell}=\frac{1}{b_{\ell}}\left(-\frac{c_{\ell-1}}{r_{\ell-1}}+\lambda-a_{\ell}\right), \quad \ell=1, \ldots k .
$$

This recursion can be solved for any parameter $\lambda$, and $\lambda$ is an eigenvalue if and only if $r_{k}=r_{k, \lambda}=0$. Induction shows that for a fixed $\ell>0$ the function $\lambda \rightarrow r_{\ell, \lambda}$ is just a rational function in $\lambda$ which is analytic and increasing between its blow-ups. (In fact, it can be shown that $r_{\ell}$ is a constant multiple of $p_{\ell}(\lambda) / p_{\ell-1}(\lambda)$ where $p_{\ell}(\cdot)$ is the characteristic polynomial of the top left $\ell \times \ell$ minor of $M$.) From this it follows easily that for each $0 \leq \ell \leq k$ we can define a continuous monotone increasing function $\lambda \rightarrow \phi_{\ell, \lambda}$ which satisfies $\tan \left(\phi_{\ell, \lambda} / 2\right)=r_{\ell, \lambda}$. The function $\varphi_{\ell, \text { is }}$ inique up to translation by integer multiples of $2 \pi$. Clearly the eigenvalues of $M$ are identified by the solutions of $\phi_{k, \lambda}=0 \bmod 2 \pi$. Since $\phi_{\ell,}$, is continuous and monotone this provides a way to identify the number of eigenvalues in $\left(\lambda_{0}, \lambda_{1}\right]$ from the values $\phi_{k, \lambda_{0}}$ and $\phi_{k, \lambda_{1}}$ :

$$
\#\left\{\left(\phi_{k, \lambda_{0}}, \phi_{k, \lambda_{1}}\right] \cap 2 \pi \mathbb{Z}\right\}=\#\left\{\text { eigenvalues in }\left(\lambda_{0}, \lambda_{1}\right]\right\}
$$


This is basically a discrete version of the Sturm-Liouville oscillation theory. (Note that if we shift $\phi_{k \text {, }}$ by $2 \pi$ then the expression on the right stays the same, so it does not matter which realization of $\phi_{k, \text {. we take.) }}$

We do not need to fully solve the recursion (19) in order to count eigenvalues. If we consider the reversed version of (19) started from index $k$ with initial condition 0 :

$$
r_{k}^{\odot}=0, \quad r_{\ell-1}^{\odot}=-c_{\ell}\left(a_{\ell}-\lambda+b_{\ell} r_{\ell}^{\odot}\right)^{-1}, \quad \ell=1, \ldots k .
$$

then $\lambda$ is an eigenvalue if and only if $r_{\ell, \lambda}=r_{\ell, \lambda}^{\odot}$. Moreover, we can turn $r_{\ell, \lambda}^{\odot}$ into an angle $\phi_{\ell, \lambda}^{\odot}$ which will be continuous and monotone decreasing in $\lambda$ (similarly as before for $r$ and $\phi$ ) which transforms the previous condition to $\phi_{\ell, \lambda}-\phi_{\ell, \lambda}^{\odot}=0 \bmod 2 \pi$. This means that we can also count eigenvalues in the interval $\left(\lambda_{0}, \lambda_{1}\right]$ by the formula

$$
\#\left\{\left(\phi_{\ell, \lambda_{0}}-\phi_{\ell, \lambda_{0}}^{\odot}, \phi_{\ell, \lambda_{1}}-\phi_{\ell, \lambda_{1}}^{\odot}\right] \cap 2 \pi \mathbb{Z}\right\}=\#\left\{\text { eigenvalues in }\left(\lambda_{0}, \lambda_{1}\right]\right\}
$$

If $h: \mathbb{R} \rightarrow \mathbb{R}$ is a monotone increasing continuous function with $h(x+2 \pi)=h(x)$ then the solutions of $\phi_{\ell, \lambda}=\phi_{\ell, \lambda}^{\odot} \bmod 2 \pi$ will be the same as that of $h\left(\phi_{\ell, \lambda}\right)=h\left(\phi_{\ell, \lambda}^{\odot}\right) \bmod 2 \pi$. Since $h\left(\phi_{\ell, \lambda}\right)-h\left(\phi_{\ell, \lambda}^{\odot}\right)$ is also continuous and increasing we get

$$
\#\left\{\left(h\left(\phi_{\ell, \lambda_{0}}\right)-h\left(\phi_{\ell, \lambda_{0}}^{\odot}\right), h\left(\phi_{\ell, \lambda_{1}}\right)-h\left(\phi_{\ell, \lambda_{1}}^{\odot}\right)\right] \cap 2 \pi \mathbb{Z}\right\}=\#\left\{\text { eigenvalues in }\left(\lambda_{0}, \lambda_{1}\right]\right\} \text {. }
$$

In our case, by analyzing the scaling limit of $h\left(\phi_{\ell,}\right)$ and $h\left(\phi_{\ell, \cdot}^{\odot}\right)$ for a certain $\ell$ and $h$ we can identify the limiting point process. This method was used in [13] for the bulk scaling limit of the $\beta$ Hermite ensemble. An equivalent approach (via transfer matrices) was used in [6] and [14] to analyze the asymptotic behavior of the spectrum for certain discrete random Schrödinger operators.

\section{The main steps of the proof}

The proof will be similar to one given for Theorem 1 in [13]. The basic idea is simple to explain: we will use (22) with a certain $\ell=\ell(n)$ and $h$. Then we will show that the length of the interval on the left hand side of the equation converges to $2 \pi\left(N\left(\lambda_{1}\right)-N\left(\lambda_{0}\right)\right)$ while the left endpoint of that interval becomes uniform modulo $2 \pi$. Since $N\left(\lambda_{1}\right)-N\left(\lambda_{0}\right)$ is a.s. integer the number of eigenvalues in $\left(\lambda_{0}, \lambda_{1}\right]$ converges to $N\left(\lambda_{1}\right)-N\left(\lambda_{0}\right)$ which shows that the scaling limit of the eigenvalue process is given by Sine $_{\beta}$.

The actual proof will require several steps. In order to limit the size of this paper and not to make it overly technical, we will recycle some parts of the proof in [13]. Our aim is to give full details whenever there is a major difference between the two proofs and to provide an outline of the proof if one can adapt parts of [13] easily.

Proof of Theorem 9 Recall that $\Lambda_{n}$ denotes the multi-set of eigenvalues for the matrix $\tilde{A}_{n, m}$ which is defined in (6). We denote by $N_{n}(\lambda)$ the counting function of the scaled random multi-sets $4 n_{0}^{1 / 2}\left(\Lambda_{n}-\right.$ $\left.\mu_{n}\right)$, we will prove that for any $\left(\lambda_{1}, \cdots, \lambda_{d}\right) \in \mathbb{R}^{d}$ we have

$$
\left(N_{n}\left(\lambda_{1}\right), \cdots, N_{n}\left(\lambda_{d}\right)\right) \stackrel{d}{\Longrightarrow}\left(N\left(\lambda_{1}\right), \cdots, N\left(\lambda_{d}\right)\right) .
$$


where $N(\lambda)=\frac{1}{2 \pi} \lim _{t \rightarrow 1} \alpha_{\lambda}(t)$ as defined using the SDE (17).

We will use the ideas described in Subsection 2.4 to analyze the eigenvalue equation $\tilde{A}_{n, m} x=\Lambda x$, where $x \in \mathbb{R}^{2 n}$. Following the scaling given in (11) we set

$$
\Lambda=\mu_{n}+\frac{\lambda}{4 \sqrt{n_{0}}} .
$$

In Section 4 we will define the regularized phase function $\varphi_{\ell, \lambda}$ and target phase function $\varphi_{\ell, \lambda}^{\odot}$ for $\ell \in\left[0, n_{0}\right)$. These will be independent of each other for a fixed $\ell$ (as functions in $\lambda$ ) and satisfy the following identity for $\lambda<\lambda^{\prime}$ :

$$
\#\left\{\left(\varphi_{\ell, \lambda}-\varphi_{\ell, \lambda}^{\odot}, \varphi_{\ell, \lambda^{\prime}}-\varphi_{\ell, \lambda^{\prime}}^{\odot}\right] \cap 2 \pi \mathbb{Z}\right\}=N_{n}\left(\lambda^{\prime}\right)-N_{n}(\lambda) .
$$

The functions $\varphi_{\ell, \lambda}$ and $\varphi_{\ell, \lambda}^{\odot}$ will be transformed versions of the phase function and target phase function $\phi_{\ell, \lambda}$ and $\phi_{\ell, \lambda}^{\odot}$ so $(24)$ will be just an application of $(22)$. The regularization is needed in order to have a version of the phase function which is asymptotically continuous. Indeed, in Proposition 17 of Section 5 we will show that for any $0<\varepsilon<1$ the rescaled version of the phase function $\varphi_{\ell, \lambda}$ in $\left[0, n_{0}(1-\varepsilon)\right]$ converges to a one-parameter family of stochastic differential equations. Moreover we will prove that in the same region the relative phase function $\alpha_{\ell, \lambda}=\varphi_{\ell, \lambda}-\varphi_{\ell, 0}$ will converge to the solution $\alpha_{\lambda}$ of the SDE (17)

$$
\alpha_{\left\lfloor n_{0}(1-\varepsilon)\right\rfloor, \lambda} \stackrel{d}{\Longrightarrow} \alpha_{\lambda}(1-\varepsilon), \quad \text { as } n \rightarrow \infty
$$

in the sense of finite dimensional distributions in $\lambda$. This will be the content of Corollary 18 .

Next we will describe the asymptotic behavior of the phase functions $\varphi_{\ell, \lambda}, \alpha_{\ell, \lambda}$ and $\varphi_{\ell, \lambda}^{\odot}$ in the stretch $\ell \in\left[\left\lfloor n_{0}(1-\varepsilon)\right\rfloor, n_{2}\right]$ where

$$
n_{2}=\left\lfloor n_{0}-\mathscr{K}\left(n_{1}^{1 / 3} \vee 1\right)\right\rfloor .
$$

(The constants $\varepsilon, \mathscr{K}$ will be determined later.) We will show that if the relative phase function is already close to an integer multiple of $2 \pi$ at $\left\lfloor n_{0}(1-\varepsilon)\right\rfloor$ then it will not change too much in the interval $\left[\left\lfloor n_{0}(1-\varepsilon)\right\rfloor, n_{2}\right]$. To be more precise, in Proposition 19 of Section 6 we will prove that there exists a constant $c=c(\bar{\lambda}, \beta)$ so that we have

$$
\mathbb{E}\left[\left|\alpha_{\left\lfloor n_{0}(1-\varepsilon)\right\rfloor, \lambda}-\alpha_{n_{2}, \lambda}\right| \wedge 1\right] \leq c\left[\operatorname{dist}\left(\alpha_{\left\lfloor n_{0}(1-\varepsilon)\right\rfloor, \lambda}, 2 \pi \mathbb{Z}\right)+\sqrt{\epsilon}+n_{0}^{-1 / 2}\left(n_{1}^{1 / 6} \vee \log n_{0}\right)+\mathscr{K}^{-1}\right]
$$

for all $\mathscr{K}>0, \epsilon \in(0,1), \lambda \leq|\bar{\lambda}|$. Note that we already know that $\alpha_{\left\lfloor n_{0}(1-\varepsilon)\right\rfloor}$ converges to $\alpha_{\lambda}(1-\varepsilon)$ in distribution (as $n \rightarrow \infty$ ) and that $\alpha_{\lambda}(1-\varepsilon$ ) converges a.s. to an integer multiple of $2 \pi$ (as $\varepsilon \rightarrow 0$ ). By the conditions on $n_{0}, n_{1}$ the term $n_{0}^{-1 / 2}\left(n_{1}^{1 / 6} \vee \log n_{0}\right)$ converges to 0 .

We will also show that if $\mathscr{K} \rightarrow \infty$ and $\mathscr{K} n_{0}^{-1}\left(n_{1}^{1 / 3} \vee 1\right) \rightarrow 0$ then the random angle $\varphi_{n_{2}, 0}$ becomes uniformly distributed modulo $2 \pi$ as $n \rightarrow \infty$ (see Proposition 23 of Section 7).

Next we will prove that the target phase function will loose its dependence on $\lambda$ : for every $\lambda \in \mathbb{R}$ and $\mathscr{K}>0$ we have

$$
\alpha_{n_{2}, \lambda}^{\odot}=\varphi_{n_{2}, \lambda}^{\odot}-\varphi_{n_{2}, 0}^{\odot} \stackrel{P}{\longrightarrow} 0, \quad \text { as } n \rightarrow \infty .
$$

This will be the content of Proposition 24 in Section 7. 
The proof now can be finished exactly the same way as in [13]. Using the previous statements and a standard diagonal argument we can choose $\varepsilon=\varepsilon(n) \rightarrow 0$ and $\mathscr{K}=\mathscr{K}(n) \rightarrow \infty$ so that the following limits all hold simultaneously:

$$
\begin{aligned}
\left(\alpha_{\left\lfloor n_{0}(1-\varepsilon)\right\rfloor, \lambda_{i}}, i=1, \ldots, d\right) & \stackrel{d}{\longrightarrow}\left(2 \pi N\left(\lambda_{i}\right), i=1, \ldots, d\right), \\
\varphi_{n_{2}, 0} & \stackrel{P}{\longrightarrow} \text { Uniform }[0,2 \pi] \quad \text { modulo } 2 \pi, \\
\alpha_{\left\lfloor n_{0}(1-\varepsilon)\right\rfloor, \lambda_{i}}-\alpha_{n_{2}, \lambda_{i}} & \stackrel{P}{\longrightarrow} 0, \quad i=1, \ldots, d, \\
\alpha_{n_{2}, \lambda_{i}}^{\odot} & \stackrel{P}{\longrightarrow} 0, \quad i=1, \ldots, d .
\end{aligned}
$$

This means that if we apply the identity (24) with $\lambda=0, \lambda^{\prime}=\lambda_{i}$ and $\ell=n_{2}$ then the length of the random intervals

$$
I_{i}=\left(\varphi_{n_{2}, 0}-\varphi_{n_{2}, 0}^{\odot}, \varphi_{n_{2}, \lambda_{i}}-\varphi_{n_{2}, \lambda_{i}}^{\odot}\right]
$$

converge to $2 \pi N\left(\lambda_{i}\right)$ in distribution (jointly), while the common left endpoint of these intervals becomes uniform modulo $2 \pi$. (Since $\varphi_{n_{2}, 0}$ and $\varphi_{n_{2}, 0}^{\odot}$ are independent and $\varphi_{n_{2}, 0}$ converges to a uniform distribution mod $2 \pi$.) This means that $\#\left\{2 k \pi \in I_{i}: k \in \mathbb{Z}\right\}$ converges to $N\left(\lambda_{i}\right)$ which proves (23) and Theorem 9 .

The following figure gives an overview of the main components of the proof.

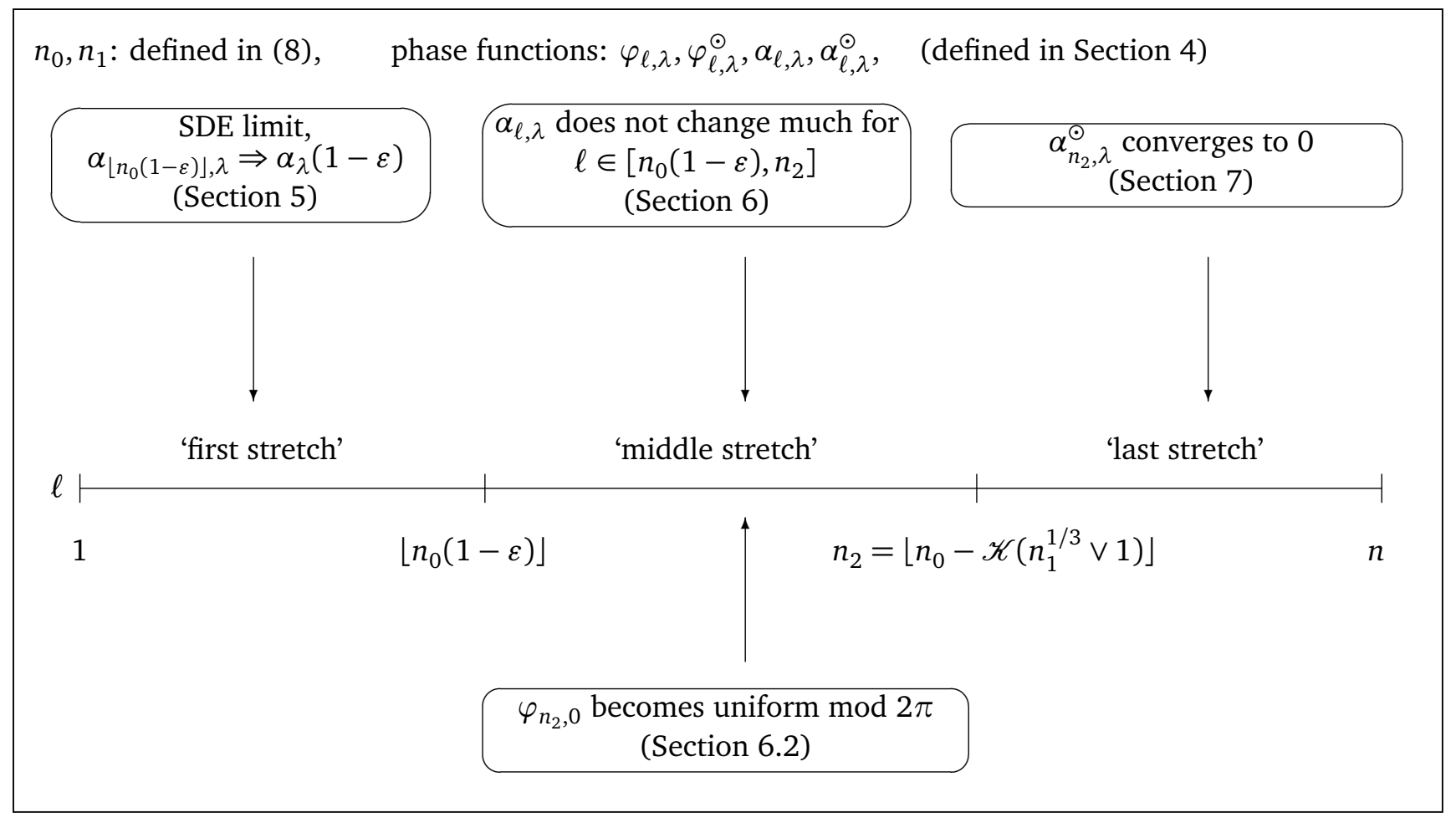

Figure 1: Outline of the proof of Theorem 9 


\section{Phase functions}

In this section we introduce the phase functions used to count the eigenvalues.

\subsection{The eigenvalue equations}

Let $s_{j}=\sqrt{n-j-1 / 2}$ and $p_{j}=\sqrt{m-j-1 / 2}$. Conjugating the matrix $\tilde{A}_{n, m}(6)$ with a $(2 n) \times(2 n)$ diagonal matrix $D=D^{(n)}$ with diagonal elements

$$
D_{1,1}=1, \quad D_{2 i, 2 i}=\frac{\tilde{\chi}_{\beta(m-i-1)}}{\sqrt{\beta} p_{i}} \prod_{\ell=1}^{i-1} \frac{\tilde{\chi}_{\beta(m-\ell)} \chi_{\beta(n-\ell)}}{\beta p_{\ell} s_{\ell}}, \quad D_{2 i+1,2 i+1}=\prod_{\ell=1}^{i} \frac{\tilde{\chi}_{\beta(m-\ell)} \chi_{\beta(n-\ell)}}{\beta p_{\ell} s_{\ell}}
$$

we get the tridiagonal matrix $\tilde{A}_{n, m}^{D}=D^{-1} \tilde{A}_{n, m} D$ :

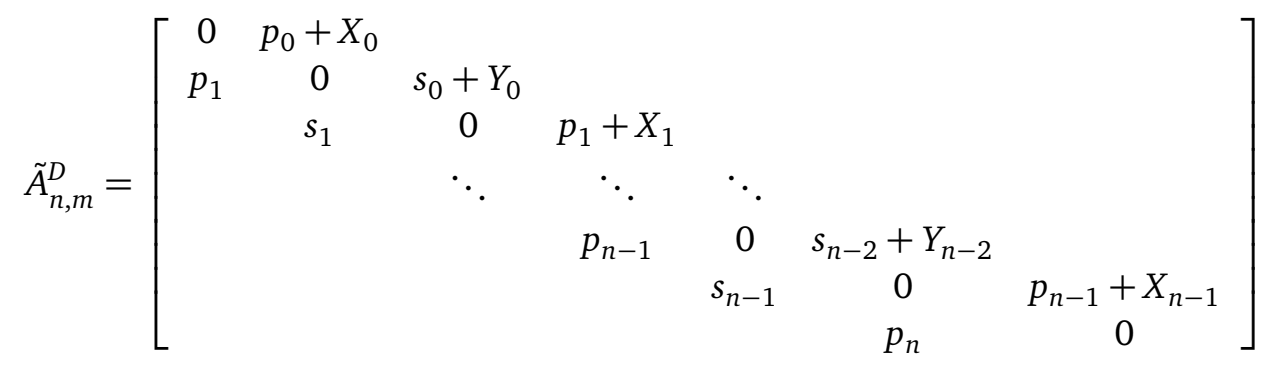

where

$$
X_{\ell}=\frac{\tilde{\chi}_{\beta(m-\ell-1)}^{2}}{\beta p_{\ell+1}}-p_{\ell}, \quad 0 \leq \ell \leq n-1, \quad Y_{\ell}=\frac{\chi_{\beta(n-\ell-1)}^{2}}{\beta s_{\ell+1}}-s_{\ell}, \quad 0 \leq \ell \leq n-2 .
$$

The first couple of moments of these random variables are explicitly computable using the moment generating function of the $\chi^{2}$-distribution and we get the following asymptotics:

$$
\begin{aligned}
& \mathbb{E} X_{\ell}=\mathscr{O}\left((m-\ell)^{-3 / 2}\right), \quad \mathbb{E} X_{\ell}^{2}=2 / \beta+\mathscr{O}\left((m-\ell)^{-1}\right), \quad \mathbb{E} X_{\ell}^{4}=\mathscr{O}(1), \\
& \mathbb{E} Y_{\ell}=\mathscr{O}\left((n-\ell)^{-3 / 2}\right), \quad \mathbb{E} Y_{\ell}^{2}=2 / \beta+\mathscr{O}\left((n-\ell)^{-1}\right), \quad \mathbb{E} Y_{\ell}^{4}=\mathscr{O}(1),
\end{aligned}
$$

where the constants in the error terms only depend on $\beta$.

We consider the eigenvalue equation for $\tilde{A}_{n, m}^{D}$ with a given $\Lambda \in \mathbb{R}$ and denote a nontrivial solution of the first $2 n-1$ components by $u_{1}, v_{1}, u_{2}, v_{2}, \ldots, u_{n}, v_{n}$. Then we have

$$
\begin{aligned}
s_{\ell} v_{\ell}+\left(p_{\ell}+X_{\ell}\right) v_{\ell+1} & =\Lambda u_{\ell+1}, & & 0 \leq \ell \leq n-1, \\
p_{\ell+1} u_{\ell+1}+\left(s_{\ell}+Y_{\ell}\right) u_{\ell+2} & =\Lambda v_{\ell+1}, & & 0 \leq \ell \leq n-2,
\end{aligned}
$$

where we set $v_{0}=0$ and we can assume $u_{1}=1$ by linearity. We set $r_{\ell}=r_{\ell, \Lambda}=u_{\ell+1} / v_{\ell}, 0 \leq \ell \leq n-1$ and $\hat{r}_{\ell}=\hat{r}_{\ell, \Lambda}=v_{\ell} / u_{\ell}, 1 \leq \ell \leq n$. These are elements of $\mathbb{R} \cup\{\infty\}$ satisfying the recursion

$$
\begin{aligned}
& \hat{r}_{\ell+1}=\left(-\frac{1}{r_{\ell}} \cdot \frac{s_{\ell}}{p_{\ell}}+\frac{\Lambda}{p_{\ell}}\right)\left(1+\frac{X_{\ell}}{p_{\ell}}\right)^{-1}, \quad 0 \leq \ell \leq n-1 \\
& r_{\ell+1}=\left(-\frac{1}{\hat{r}_{\ell+1}} \cdot \frac{p_{\ell+1}}{s_{\ell}}+\frac{\Lambda}{s_{\ell}}\right)\left(1+\frac{Y_{\ell}}{s_{\ell}}\right)^{-1}, \quad 0 \leq \ell \leq n-2,
\end{aligned}
$$

with initial condition $r_{0}=\infty$. We can set $Y_{n}=0$ and define $r_{n}$ via (32) with $\ell=n-1$, then $\Lambda$ is an eigenvalue if and only if $r_{n}=0$. 


\subsection{The hyperbolic point of view}

We use the hyperbolic geometric approach of [13] to study the evolution of $r$ and $\hat{r}$. We will view $\mathbb{R} \cup\{\infty\}$ as the boundary of the hyperbolic plane $\mathbb{H}=\{\mathfrak{I} z>0: z \in \mathbb{C}\}$ in the Poincaré half-plane model. We denote the group of linear fractional transformations preserving $\mathbb{H}$ by $\operatorname{PSL}(2, \mathbb{R})$. The recursions for both $r$ and $\hat{r}$ evolve by elements of this group of the form $x \mapsto b-a / x$ with $a>0$.

The Poincaré half-plane model is equivalent to the Poincaré disk model $\mathbb{U}=\{|z|<1\}$ via the conformal bijection $\mathbf{U}(z)=\frac{i z+1}{z+i}$ which is also a bijection between the boundaries $\partial \mathbb{H}=\mathbb{R} \cup\{\infty\}$ and $\partial \mathbb{U}=\{|z|=1, z \in \mathbb{C}\}$. Thus elements of $\operatorname{PSL}(2, \mathbb{R})$ also act naturally on the unit circle $\partial \mathbb{U}$. By lifting these maps to $\mathbb{R}$, the universal cover of $\partial \mathbb{U}$, each element $T$ in $\operatorname{PSL}(2, \mathbb{R})$ becomes an $\mathbb{R} \rightarrow \mathbb{R}$ function. The lifted versions are uniquely determined up to shifts by $2 \pi$ and will also form a group which we denote by $\operatorname{UPSL}(2, \mathbb{R})$. For any $\mathbf{T} \in \operatorname{UPSL}(2, \mathbb{R})$ we can look at $\mathbf{T}$ as a function acting on $\partial \mathbb{H}, \partial \mathbb{U}$ or $\mathbb{R}$. We will denote these actions by:

$$
\partial \mathbb{H} \rightarrow \partial \mathbb{H}: z \mapsto z . \mathbf{T}, \quad \partial \mathbb{U} \rightarrow \partial \mathbb{U}: z \mapsto z \mathbf{T}, \quad \partial \mathbb{R} \rightarrow \partial \mathbb{R}: z \mapsto z_{*} \mathbf{T} .
$$

For every $\mathbf{T} \in \operatorname{UPSL}(2, \mathbb{R})$ the function $x \mapsto f(x)=x_{*} T$ is monotone, analytic and quasiperiodic modulo $2 \pi: f(x+2 \pi)=f(x)+2 \pi$. It is clear from the definitions that $e^{i x}{ }_{\circ} T=e^{i f(x)}$ and $(2 \tan (x)) \cdot \mathbf{T}=2 \tan f(x)$.

Now we will introduce a couple of simple elements of UPSL(2, $\mathbb{R})$. For a given $\alpha \in \mathbb{R}$ we will denote by $\mathbf{Q}(\alpha)$ the rotation by $\alpha$ in $\mathbb{U}$ about 0 . More precisely, $\varphi_{*} \mathbf{Q}(\alpha)=\varphi+\alpha$. For $a>0, b \in \mathbb{R}$ we denote by $\mathbf{A}(a, b)$ the affine map $z \rightarrow a(z+b)$ in $\mathbb{H}$. This is an element of $\operatorname{PSL}(2, \mathbb{R})$ which fixes $\infty$ in $\mathbb{H}$ and -1 in $\partial \mathbb{U}$. We specify its lifted version in $\operatorname{UPSL}(2, \mathbb{R})$ by making it fix $\pi$, this will uniquely determines it as a $\mathbb{R} \rightarrow \mathbb{R}$ function.

Given $\mathrm{T} \in \operatorname{UPSL}(2, \mathbb{R}), x, y \in \mathbb{R}$ we define the angular shift

$$
\operatorname{ash}(\mathbf{T}, x, y)=(y * \mathbf{T}-x * \mathbf{T})-(y-x)
$$

which gives the change in the signed distance of $x, y$ under $\mathbf{T}$. This only depends on $v=e^{i x}, w=e^{i y}$ and the effect of $\mathbf{T}$ on $\partial U$, so we can also view $\operatorname{ash}(\mathbf{T}, \cdot, \cdot)$ as a function on $\partial U \times \partial U$ and the following identity holds:

$$
\operatorname{ash}(\mathbf{T}, v, w)=\arg _{[0,2 \pi)}\left(w_{\circ} \mathbf{T} / v_{\circ} \mathbf{T}\right)-\arg _{[0,2 \pi)}(w / v) .
$$

The following lemma appeared as Lemma 16 in [13], it provides a useful estimate for the angular shift.

Lemma 12. Suppose that for a $\mathbf{T} \in \operatorname{UPSL}(2, \mathbb{R})$ we have $(i+z) . \mathbf{T}=i$ with $|z| \leq \frac{1}{3}$. Then

$$
\begin{aligned}
\operatorname{ash}(\mathbf{T}, v, w) & =\Re\left[(\bar{w}-\bar{v})\left(-z-\frac{i(2+\bar{v}+\bar{w})}{4} z^{2}\right)\right]+\varepsilon_{3} \\
& =-\Re[(\bar{w}-\bar{v}) z]+\varepsilon_{2}=\varepsilon_{1},
\end{aligned}
$$

where for $d=1,2,3$ and an absolute constant $c$ we have

$$
\left|\varepsilon_{d}\right| \leq c|w-v||z|^{d} \leq 2 c|z|^{d} .
$$

If $v=-1$ then the previous bounds hold even in the case $|z|>\frac{1}{3}$. 


\subsection{Regularized phase functions}

Because of the scaling in (11) we will set

$$
\Lambda=\mu_{n}+\frac{\lambda}{4 n_{0}^{1 / 2}}
$$

We introduce the following operators

$$
\begin{array}{ll}
\mathbf{J}_{\ell}=\mathbf{Q}(\pi) \mathbf{A}\left(s_{\ell} / p_{\ell}, \mu_{n} / s_{\ell}\right), & \mathbf{M}_{\ell}=\mathbf{A}\left(\left(1+X_{\ell} / p_{\ell}\right)^{-1}, \lambda /\left(4 n_{0}^{1 / 2} p_{\ell}\right)\right) \mathbf{A}\left(\frac{p_{\ell}}{p_{\ell+1}}, 0\right), \\
\hat{\mathbf{J}}_{\ell}=\mathbf{Q}(\pi) \mathbf{A}\left(p_{\ell} / s_{\ell}, \mu_{n} / p_{\ell}\right), & \hat{\mathbf{M}}_{\ell}=\mathbf{A}\left(\left(1+Y_{\ell} / s_{\ell}\right)^{-1}, \lambda /\left(4 n_{0}^{1 / 2} s_{\ell}\right)\right) .
\end{array}
$$

Then (31) and (32) can be rewritten as

$$
r_{\ell+1}=r_{\ell} \cdot \mathbf{J}_{\ell} \mathbf{M}_{\ell} \hat{\mathbf{J}}_{\ell} \hat{\mathbf{M}}_{\ell}, \quad r_{0}=\infty .
$$

(We suppressed the $\lambda$ dependence in $r$ and the operators $\mathbf{M}, \hat{\mathbf{M}}$.) Lifting these recursions from $\partial \mathbb{H}$ to $\mathbb{R}$ we get the evolution of the corresponding phase angle which we denote by $\phi_{\ell}=\phi_{\ell, \lambda}$.

$$
\phi_{\ell+1}=\phi_{\ell * \mathbf{J}_{\ell}} \mathbf{M}_{\ell} \hat{\mathbf{J}}_{\ell} \hat{\mathbf{M}}_{\ell}, \quad \phi_{0}=-\pi .
$$

Solving the recursion from the other end, with end condition 0 we get the target phase function $\phi_{\ell, \lambda}^{\odot}$ :

$$
\phi_{\ell}^{\odot}=\phi_{\ell+1}^{\odot} * \hat{\mathbf{M}}_{\ell}^{-1} \hat{\mathbf{J}}_{\ell}^{-1} \mathbf{M}_{\ell}^{-1} \mathbf{J}_{\ell}^{-1}, \quad \phi_{n}^{\odot}=0 .
$$

It is clear that $\phi_{\ell, \lambda}$ and $\phi_{\ell, \lambda}^{\odot}$ are independent for a fixed $\ell$ (as functions in $\lambda$ ), they are monotone and analytic in $\lambda$ and we can count eigenvalues using the formula (21).

In our case both $\mathbf{M}_{\ell}$ and $\hat{\mathbf{M}}_{\ell}$ will be small perturbations of the identity so $\mathbf{J}_{\ell} \hat{\mathbf{J}}_{\ell}$ will be the main part of the evolution. This is a rotation in the hyperbolic plane if it only has one fixed point in $\mathbb{H}$. The fixed point equation $\rho_{\ell}=\rho_{\ell} \cdot \mathbf{J}_{\ell} \hat{\mathbf{J}}_{\ell}$ can be rewritten as

$$
\rho_{\ell}=\frac{p_{\ell}}{s_{\ell}}\left(\frac{\mu_{n}}{p_{\ell}}-\frac{p_{\ell}}{\left(\frac{\mu_{n}}{s_{\ell}}-\frac{1}{\rho_{\ell}}\right)}\right)=\frac{\rho_{\ell}\left(\mu_{n}^{2}-p_{\ell}^{2}\right)-\mu_{n} s_{\ell}}{\rho_{\ell} \mu_{n} s_{\ell}-s_{\ell}^{2}} .
$$

This can be solved explicitly, and one gets the following unique solution in the upper half plane if $\ell<n_{0}+1 / 2$ :

$$
\rho_{\ell}=\frac{\mu_{n}^{2}-m+n}{2 \mu_{n} s_{\ell}}+i \sqrt{1-\frac{\left(\mu_{n}^{2}-m+n\right)^{2}}{4 \mu_{n}^{2} s_{\ell}^{2}}} .
$$

One also needs to use the identity $p_{\ell}^{2}-s_{\ell}^{2}=m-n$ and $(12)$. This shows that if $\ell<n_{0}$ then $\mathbf{J}_{\ell} \hat{\mathbf{J}}_{\ell}$ is a rotation in the hyperbolic plane. We can move the center of rotation to 0 in $\mathbb{U}$ by conjugating it with an appropriate affine transformation:

$$
\mathbf{J}_{\ell} \hat{\mathbf{J}}_{\ell}=\mathbf{Q}\left(-2 \arg \left(\rho_{\ell} \hat{\rho}_{\ell}\right)\right)^{\mathbf{T}_{\ell}^{-1}} .
$$

Here $\mathbf{T}_{\ell}=\mathbf{A}\left(\mathfrak{I}\left(\rho_{\ell}\right)^{-1},-\Re \rho_{\ell}\right), \mathbf{X}^{\mathbf{Y}}=\mathbf{Y}^{-1} \mathbf{X Y}$ and

$$
\hat{\rho}_{\ell}=\frac{\mu_{n}^{2}+m-n}{2 \mu_{n} p_{\ell}}+i \sqrt{1-\frac{\left(\mu_{n}^{2}+m-n\right)^{2}}{4 \mu_{n}^{2} p_{\ell}^{2}}} .
$$


In order to regularize the evolution of the phase function we introduce

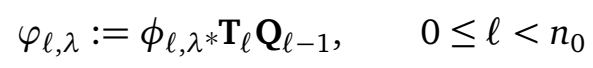

where $\mathbf{Q}_{\ell}=\mathbf{Q}\left(2 \arg \left(\rho_{0} \hat{\rho}_{0}\right)\right) \cdots \mathbf{Q}\left(2 \arg \left(\rho_{\ell} \hat{\rho}_{\ell}\right)\right)$ and $\mathbf{Q}_{-1}$ is the identity. It is easy to check that the initial condition remains $\varphi_{0, \lambda}=\pi$. Then

$$
\begin{aligned}
\varphi_{\ell+1} & =\varphi_{\ell^{*}} \mathbf{Q}_{\ell-1}^{-1} \mathbf{T}_{\ell}^{-1} \mathbf{J}_{\ell} \mathbf{M}_{\ell} \hat{\mathbf{J}}_{\ell} \hat{\mathbf{M}}_{\ell} \mathbf{T}_{\ell+1} \mathbf{Q}_{\ell} \\
& =\varphi_{\ell^{*}} \mathbf{Q}_{\ell-1}^{-1} \mathbf{T}_{\ell}^{-1} \mathbf{Q}\left(-2 \arg \left(\rho_{\ell}\right)\right)^{\mathbf{T}_{\ell}^{-1}} \mathbf{M}_{\ell}^{\hat{\mathbf{j}}_{\ell}} \hat{\mathbf{M}}_{\ell} \mathbf{T}_{\ell} \mathbf{T}_{\ell}^{-1} \mathbf{T}_{\ell+1} \mathbf{Q}_{\ell} \\
& =\varphi_{\ell^{*}}\left(\left(\mathbf{M}_{\ell}^{\hat{\mathbf{J}}_{\ell}}\right)^{\mathbf{T}_{\ell}}\right)^{\mathbf{Q}_{\ell}}\left(\left(\hat{\mathbf{M}}_{\ell}\right)^{\mathbf{T}_{\ell}}\right)^{\mathbf{Q}_{\ell}}\left(\mathbf{T}_{\ell}^{-1} \mathbf{T}_{\ell+1}\right)^{\mathbf{Q}_{\ell}}
\end{aligned}
$$

Note that the evolution operator is now infinitesimal: $\mathbf{M}_{\ell}, \hat{\mathbf{M}}_{\ell}$ and $\mathbf{T}_{\ell}^{-1} \mathbf{T}_{\ell+1}$ will all be asymptotically small, and the various conjugations will not change this.

We can also introduce the corresponding target phase function

$$
\varphi_{\ell, \lambda}^{\odot}:=\phi_{\ell, \lambda}^{\odot} * \mathbf{T}_{\ell} \mathbf{Q}_{\ell-1}, \quad 0 \leq \ell<n_{0} .
$$

The new, regularized phase functions $\varphi_{\ell, \lambda}$ and $\varphi_{\ell, \lambda}^{\odot}$ have the same properties as $\phi, \phi^{\odot}$, i.e.: they are independent for a fixed $\ell$ (as functions in $\lambda$ ), they are monotone and analytic in $\lambda$ and we can count eigenvalues using the formula (24). (See (22) and the discussion before it.)

We will further simplify the evolution using the following identities:

$$
-\frac{a}{r}+b=\left(\frac{b^{2}+1}{a} r-b\right) \mathbf{Q}\left(\arg \left(\frac{b-i}{b+i}\right)\right), \quad r . \hat{\mathbf{J}}_{\ell} \mathbf{T}_{\ell}=-\frac{1}{r} \frac{p_{\ell}}{s_{\ell} \mathfrak{I} \rho_{\ell}}+\frac{\mu_{n}}{s_{\ell} \mathfrak{I} \rho_{\ell}}-\frac{\Re \rho_{\ell}}{\mathfrak{I} \rho_{\ell}} .
$$

From this we get

$$
\hat{\mathbf{J}}_{\ell} \mathbf{T}_{\ell}=\hat{\mathbf{T}}_{\ell} \mathbf{Q}_{\ell}\left(-2 \arg \left(\hat{\rho}_{\ell}\right)\right)
$$

where

$$
\begin{aligned}
r . \hat{\mathbf{T}}_{\ell} & =\left(\frac{s_{\ell}}{\mathfrak{I} \rho_{\ell} p_{\ell}}-2 \frac{\mathfrak{R} \rho_{\ell}}{\mathfrak{I} \rho_{\ell}} \mu_{n}+\frac{\mu_{n}^{2}}{\mathfrak{S} \rho_{\ell} p_{\ell} s_{\ell}}\right) r-\frac{\mu_{n}}{s_{\ell} \mathfrak{I} \rho_{\ell}}+\frac{\mathfrak{R} \rho_{\ell}}{\mathfrak{S} \rho_{\ell}} \\
& =\frac{1}{\mathfrak{I} \hat{\rho}_{\ell}} r-\frac{\mathfrak{A} \hat{\rho}_{\ell}}{\mathfrak{I} \hat{\rho}_{\ell}} .
\end{aligned}
$$

This allows us to write

$$
\left(\left(\mathbf{M}_{\ell}^{\hat{\mathbf{S}}_{\ell}}\right)^{\mathbf{T}_{\ell}}\right)^{\mathbf{Q}_{\ell}}=\left(\mathbf{M}_{\ell}^{\hat{\mathbf{T}}_{\ell}}\right)^{\mathbf{Q}\left(-2 \arg \left(\hat{\rho}_{\ell}\right)\right) \mathbf{Q}_{\ell}}=\left(\mathbf{M}_{\ell}^{\hat{\mathbf{T}}_{\ell}}\right)^{\hat{\mathbf{Q}}_{\ell}} .
$$

where

$$
\hat{\mathbf{Q}}_{\ell}=\mathbf{Q}_{\ell} \mathbf{Q}\left(-2 \arg \left(\hat{\rho}_{\ell}\right)\right) .
$$

Thus

$$
\varphi_{\ell+1}=\varphi_{\ell^{*}}\left(\mathbf{M}_{\ell}^{\hat{\mathbf{T}}_{\ell}}\right)^{\hat{\mathbf{Q}}_{\ell}}\left(\hat{\mathbf{M}}_{\ell}^{\mathbf{T}_{\ell}}\right)^{\mathbf{Q}_{\ell}}\left(\mathbf{T}_{\ell}^{-1} \mathbf{T}_{\ell+1}\right)^{\mathbf{Q}_{\ell}} .
$$


We will introduce the following operators to break up the evolution into smaller pieces:

$$
\begin{aligned}
& \mathbf{L}_{\ell, \lambda}=\mathbf{A}\left(1, \lambda /\left(4 n_{0}^{1 / 2} p_{\ell}\right)\right), \quad \hat{\mathbf{L}}_{\ell, \lambda}=\mathbf{A}\left(1, \lambda /\left(4 n_{0}^{1 / 2} s_{\ell}\right)\right), \\
& \mathbf{s}_{\ell, \lambda}=\mathbf{L}_{\ell, \lambda}^{\hat{\mathbf{T}}_{\ell}}\left(\hat{\mathbf{T}}_{\ell}^{-1} \mathbf{A}\left(\frac{p_{\ell}}{p_{\ell+1}}\left(1+X_{\ell} / p_{\ell}\right)^{-1}, 0\right) \hat{\mathbf{T}}_{\ell}\right), \\
& \hat{\mathbf{S}}_{\ell, \lambda}=\hat{\mathbf{L}}_{\ell, \lambda}^{\mathbf{T}_{\ell}}\left(\mathbf{T}_{\ell}^{-1} \mathbf{A}\left(\left(1+Y_{\ell} / s_{\ell}\right)^{-1}, 0\right) \mathbf{T}_{\ell+1}\right) .
\end{aligned}
$$

Then

$$
\varphi_{\ell+1}=\varphi_{\ell^{*}}\left(\mathbf{L}_{\ell}^{\hat{\mathbf{T}}_{\ell}}\right)^{\hat{\mathbf{Q}}_{\ell}}\left(\mathbf{S}_{\ell, 0}\right)^{\hat{\mathbf{Q}}_{\ell}}\left(\hat{\mathbf{L}}_{\ell}^{\mathbf{T}_{\ell}}\right)^{\mathbf{Q}_{\ell}}\left(\hat{\mathbf{S}}_{\ell, 0}\right)^{\mathbf{Q}_{\ell}}=\varphi_{\ell^{*}}\left(\mathbf{S}_{\ell, \lambda}\right)^{\hat{\mathbf{Q}}_{\ell}}\left(\hat{\mathbf{S}}_{\ell, \lambda}\right)^{\mathbf{Q}_{\ell}}
$$

We also introduce the relative (regularized) phase function and target phase function:

$$
\alpha_{\ell, \lambda}:=\varphi_{\ell, \lambda}-\varphi_{\ell, 0}, \quad \alpha_{\ell, \lambda}^{\odot}:=\varphi_{\ell, \lambda}^{\odot}-\varphi_{\ell, 0}^{\odot} .
$$

\section{SDE limit for the phase function}

Let $\mathscr{F}_{\ell}$ denote the $\sigma$-field generated by $\varphi_{j, \lambda}, j \leq \ell-1$. Then $\varphi_{\ell, \lambda}$ is a Markov chain in $\ell$ with respect to $\mathscr{F}_{\ell}$. Indeed, the relation (42) shows that $\varphi_{\ell+1, \lambda}=h_{\ell, \lambda}\left(\varphi_{\ell+1, \lambda}, X_{\ell}, Y_{\ell}\right)$ where $h_{\ell, \lambda}$ is a deterministic function depending on $\ell$ and $\lambda$. Since $X_{\ell}, Y_{\ell}$ are independent of $\mathscr{F}_{\ell}$ it follows that $\mathbb{E}\left[\varphi_{\ell+1, \lambda} \mid \mathscr{F}_{\ell}\right]=\mathbb{E}\left[\varphi_{\ell+1, \lambda} \mid \varphi_{\ell, \lambda}\right]$. We will show that this Markov chain converges to a diffusion limit after proper normalization. In order to do this we will estimate $\mathbb{E}\left[\varphi_{\ell+1, \lambda}-\varphi_{\ell, \lambda} \mid \mathscr{F}_{\ell}\right]$ and $\mathbb{E}\left[\left(\varphi_{\ell+1, \lambda}-\varphi_{\ell, \lambda}\right)\left(\varphi_{\ell+1, \lambda^{\prime}}-\varphi_{\ell, \lambda^{\prime}}\right) \mid \mathscr{F}_{\ell}\right]$ using the angular shift lemma, Lemma 12 .

To simplify the computations we introduce 'intermediate' values for the process $\varphi_{\ell, \lambda}$ by breaking the evolution operator in (42) into two parts:

$$
\varphi_{\ell+1 / 2, \lambda}=\varphi_{\ell^{*}}\left(\mathbf{S}_{\ell, \lambda}\right)^{\hat{\mathbf{Q}}_{\ell}}, \quad \mathscr{F}_{\ell+1 / 2}=\sigma\left(\mathscr{F}_{\ell} \cup\left\{\varphi_{\ell+1 / 2, \lambda}\right\}\right) .
$$

Note that $\varphi_{\ell, \lambda}$ is still a Markov chain if we consider it as a process on the half integers.

Remark 13. We would like to note that the 'half-step' evolution rules $\varphi_{\ell, \lambda} \rightarrow \varphi_{\ell+1 / 2, \lambda}, \varphi_{\ell+1 / 2, \lambda} \rightarrow$ $\varphi_{\ell+1, \lambda}$ are very similar to the one-step evolution of the phase function $\varphi$ in [13]. Indeed, in [13], the evolution of $\varphi$ is of the type $\varphi_{\ell+1}=\varphi_{\ell^{*}}\left(\tilde{\mathbf{S}}_{\ell, \lambda}\right)^{\tilde{\mathbf{Q}}_{\ell}}$ where $\tilde{\mathbf{S}}$ is an affine transformation and $\tilde{\mathbf{Q}}$ is a rotation similar to our $\mathbf{S}, \hat{\mathbf{S}}$ and $\mathbf{Q}, \hat{\mathbf{Q}}$. In our case the evolution of $\varphi_{\ell+1, \lambda}$ is the composition of two transformations with similar structure. The main difficulties in our computations are caused by the fact that $\mathbf{Q}$ and $\mathbf{Q}$ are rather different which makes the oscillating terms more complicated.

\subsection{Single step estimates}

Throughout the rest of the proof we will use the notation $k=n_{0}-\ell$. We will need to rescale the discrete time by $n_{0}$ in order to get a limit, we will use $t=\ell / n_{0}$ and also introduce $\hat{s}(t)=\sqrt{1-t}$. 
We start with the identity

$$
\begin{aligned}
p_{\ell} \mathfrak{S} \hat{\rho}_{\ell}=s_{\ell} \mathfrak{S} \rho_{\ell} & =\sqrt{s_{\ell}^{2}-\frac{\left(\mu_{n}^{2}-m+n\right)^{2}}{4 \mu_{n}^{2}}}=\sqrt{n-\frac{\left(\mu_{n}^{2}-m+n\right)^{2}}{4 \mu_{n}^{2}}-\ell-\frac{1}{2}} \\
& =\sqrt{n_{0}-\ell}=\sqrt{k}=\sqrt{n_{0}} \hat{s}(t) .
\end{aligned}
$$

Note that this means that

$$
\begin{aligned}
& \rho_{\ell}= \pm \sqrt{\frac{n-n_{0}-1 / 2}{n-\ell-1 / 2}}+i \sqrt{\frac{n_{0}-\ell}{n-\ell-1 / 2}}= \pm \sqrt{\frac{n_{1}}{n_{1}+k}}+i \sqrt{\frac{k}{n_{1}+k}} \\
& \hat{\rho}_{\ell}=\sqrt{\frac{m-n_{0}-1 / 2}{m-\ell-1 / 2}}+i \sqrt{\frac{n_{0}-\ell}{m-\ell-1 / 2}}=\sqrt{\frac{m_{1}}{m_{1}+k}}+i \sqrt{\frac{k}{m_{1}+k}}
\end{aligned}
$$

where the sign in $\Re \rho_{\ell}$ is positive if $\mu_{n}>\sqrt{m-n}$ and negative otherwise.

For the angular shift estimates we need to consider

$$
\begin{aligned}
& Z_{\ell, \lambda}:=i . S_{\ell, \lambda}^{-1}-i=\frac{\hat{\rho}_{\ell} X_{\ell}}{\sqrt{n_{0}} \hat{s}(t)} \cdot \frac{p_{\ell+1}}{p_{\ell}}+\left(-\frac{\lambda}{4 n_{0} \hat{s}(t)}+\frac{\hat{\rho}_{\ell}\left(p_{\ell+1}-p_{\ell}\right)}{p_{\ell} \mathfrak{S} \hat{\rho}_{\ell}}\right)=: V_{\ell}+v_{\ell}, \\
& \hat{Z}_{\ell, \lambda}:=i . \hat{\mathbf{S}}_{\ell, \lambda}^{-1}-i=\frac{\rho_{\ell} Y_{\ell}}{\sqrt{n_{0}} \hat{s}(t)}+\left(-\frac{\lambda}{4 n_{0} \hat{s}(t)}+\frac{\rho_{\ell+1}-\rho_{\ell}}{\mathfrak{S} \rho_{\ell}}\right)=: \hat{V}_{\ell}+\hat{v}_{\ell} .
\end{aligned}
$$

Here $V_{\ell}, \hat{V}_{\ell}$ are the random and $v_{\ell}, \hat{v}_{\ell}$ are the deterministic parts of the appropriate expressions. We have the following estimates for the deterministic parts (by Taylor expansion):

$$
\begin{aligned}
& v_{\ell, \lambda}=\frac{v_{\lambda}(t)}{n_{0}}+O\left(k^{-2}\right), \quad v_{\lambda}(t)=-\frac{\lambda}{4 \hat{s}(t)}-\frac{\hat{\rho}(t)}{2 p(t) \hat{s}(t)}, \quad\left|v_{\ell, \lambda}\right| \leq \frac{c}{k}, \\
& \hat{v}_{\ell, \lambda}=\frac{\hat{v}_{\lambda}(t)}{n_{0}}+O\left(k^{-2}\right), \quad \hat{v}_{\lambda}(t)=-\frac{\lambda}{4 \hat{s}(t)}+\frac{\frac{d}{d t} \rho(t)}{\mathfrak{I} \rho(t)}, \quad\left|\hat{v}_{\ell, \lambda}\right| \leq \frac{c}{k},
\end{aligned}
$$

where $p(t)=p^{(n)}(t)=\sqrt{m / n_{0}-t}$ and $\rho(t)=\rho^{(n)}(t), \hat{\rho}(t)=\hat{\rho}^{n}(t)$ are defined by equations 44 and (45) with $\ell=n_{0} t$. For the random terms from (30) we get

$$
\begin{aligned}
\mathbb{E} V_{\ell} & =\mathbb{E} \hat{V}_{\ell}=\mathscr{O}\left(k^{-1 / 2}(n-\ell)^{-3 / 2}\right), \\
\mathbb{E} V_{\ell}^{2} & =\frac{1}{n_{0}} q^{(1)}(t)+\mathscr{O}\left(k^{-1}(n-\ell)^{-1}\right), \quad \mathbb{E} \hat{V}_{\ell}^{2}=\frac{1}{n_{0}} q^{(2)}(t)+\mathscr{O}\left(k^{-1}(n-\ell)^{-1}\right), \\
\mathbb{E}\left|V_{\ell}^{2}\right| & =\mathbb{E}\left|\hat{V}_{\ell}^{2}\right|=\frac{1}{n_{0}} q^{(3)}(t)+\mathscr{O}\left(k^{-1}(n-\ell)^{-1}\right), \quad \mathbb{E}\left|V_{\ell}^{d}\right|, \mathbb{E}\left|\hat{V}_{\ell}^{2}\right|=\mathscr{O}\left(k^{-d / 2}\right), d=3,4,
\end{aligned}
$$

where the constants in the error term only depend on $\beta$ and

$$
q^{(1)}(t)=\frac{2 \hat{\rho}(t)^{2}}{\beta \hat{s}(t)^{2}}, \quad q^{(2)}(t)=\frac{2 \rho(t)^{2}}{\beta \hat{s}(t)^{2}}, \quad q^{(3)}(t)=\frac{2}{\beta \hat{s}(t)^{2}} .
$$

We introduce the notations

$$
\Delta_{1 / 2} f_{x, \lambda}=f_{x+1 / 2, \lambda}-f_{x, \lambda}, \quad \Delta f_{x, \lambda}=f_{x+1, \lambda}-f_{x, \lambda}
$$

and we also set for $\ell \in \mathbb{Z}_{+}$

$$
\eta_{\ell}=\rho_{0}^{2} \hat{\rho}_{0}^{2} \rho_{1}^{2} \hat{\rho}_{1}^{2} \ldots \rho_{\ell}^{2} \hat{\rho}_{\ell}^{2} .
$$

The following proposition is the analogue of Proposition 22 in [13]. 
Proposition 14. For $\ell \leq n_{0}$ we have

$$
\begin{aligned}
& \mathbb{E}\left[\Delta_{1 / 2} \varphi_{\ell, \lambda} \mid \varphi_{\ell, \lambda}=x\right]=\frac{1}{n_{0}} b_{\lambda}^{(1)}(t)+\frac{1}{n_{0}} o s c^{(1)}+\mathscr{O}\left(k^{-3 / 2}\right)=\mathscr{O}\left(k^{-1}\right) \\
& \mathbb{E}\left[\Delta_{1 / 2} \varphi_{\ell, \lambda} \Delta_{1 / 2} \varphi_{\ell, \lambda^{\prime}} \mid \varphi_{\ell, \lambda}=x, \varphi_{\ell, \lambda^{\prime}}=y\right]=\frac{1}{n_{0}} a^{(1)}(t, x, y)+\frac{1}{n_{0}} o s c^{(2)}+\mathscr{O}\left(k^{-3 / 2}\right) \\
& \mathbb{E}\left[\Delta_{1 / 2} \varphi_{\ell+1 / 2, \lambda} \mid \varphi_{\ell+1 / 2, \lambda}=x\right]=\frac{1}{n_{0}} b_{\lambda}^{(2)}(t)+\frac{1}{n_{0}} o s c^{(3)}+\mathscr{O}\left(k^{-3 / 2}\right)=\mathscr{O}\left(k^{-1}\right) \\
& \mathbb{E}\left[\Delta_{1 / 2} \varphi_{\ell+1 / 2, \lambda} \Delta_{1 / 2} \varphi_{\ell+1 / 2, \lambda^{\prime}} \mid \varphi_{\ell+1 / 2, \lambda}=x, \varphi_{\ell+1 / 2, \lambda^{\prime}}=y\right]=\frac{1}{n_{0}} a^{(2)}(t, x, y)+\frac{1}{n_{0}} o s c^{(4)}+\mathscr{O}\left(k^{-3 / 2}\right), \\
& \mathbb{E}\left[\left|\Delta_{1 / 2} \varphi_{\ell, \lambda}\right|^{d} \mid \varphi_{\ell, \lambda}=x\right], \mathbb{E}\left[\left|\Delta_{1 / 2} \varphi_{\ell+1 / 2, \lambda}\right|^{d} \mid \varphi_{\ell, \lambda}=x\right]=\mathscr{O}\left(k^{-d / 2}\right), \quad d=2,3
\end{aligned}
$$

where $t=\ell / n_{0}$,

$$
\begin{aligned}
b_{\lambda}^{(1)}=\frac{\lambda}{4 \hat{s}}+\frac{\Re \hat{\rho}}{2 p \hat{s}}+\frac{\mathfrak{s} \hat{\rho}^{2}}{2 \beta \hat{s}^{2}}, & b_{\lambda}^{(2)}=\frac{\lambda}{4 \hat{s}}-\frac{\Re \frac{d}{d t} \rho}{\mathfrak{I} \rho}+\frac{\mathfrak{I} \rho^{2}}{2 \beta \hat{s}^{2}}, \\
a^{(1)}=\frac{1}{\beta \hat{s}^{2}} \Re\left[e^{i(x-y)}\right]+\frac{1+\Re \hat{\rho}^{2}}{\beta \hat{s}^{2}}, & a^{(2)}=\frac{1}{\beta \hat{s}^{2}} \Re\left[e^{i(x-y)}\right]+\frac{1+\Re \rho^{2}}{\beta \hat{s}^{2}} .
\end{aligned}
$$

The oscillatory terms are

$$
\begin{aligned}
& o s c^{(1)}=\Re\left(\left(-v_{\ell}-i q^{(1)} / 2\right) e^{-i x} \hat{\rho}_{\ell}^{-2} \eta_{\ell}\right)+\Re\left(i e^{-2 i x} \hat{\rho}_{\ell}^{-4} \eta_{\ell}^{2} q^{(1)}\right) / 4, \\
& o s c^{(2)}=q^{(3)} \Re\left(e^{-i x} \hat{\rho}_{\ell}^{-2} \eta_{\ell}+e^{-i y} \hat{\rho}_{\ell}^{-2} \eta_{\ell}\right) / 2+\Re\left(q^{(1)}\left(e^{-i x} \hat{\rho}_{\ell}^{-2} \eta_{\ell}+e^{-i y} \hat{\rho}_{\ell}^{-2} \eta_{\ell}+e^{-i(x+y)} \hat{\rho}_{\ell}^{-4} \eta_{\ell}^{2}\right)\right) / 2, \\
& o s c^{(3)}=\Re\left(\left(-\hat{v}_{\ell}-i q^{(2)} / 2\right) e^{-i x} \eta_{\ell}\right)+\Re\left(i e^{-2 i x} \eta_{\ell}^{2} q^{(2)}\right) / 4, \\
& o s c^{(4)}=q^{(3)} \Re\left(e^{-i x} \eta_{\ell}+e^{-i y} \eta_{\ell}\right) / 2+\Re\left(q^{(2)}\left(e^{-i x} \eta_{\ell}+e^{-i y} \eta_{\ell}+e^{-i(x+y)} \eta_{\ell}^{2}\right)\right) / 2 .
\end{aligned}
$$

Proof. We start with the identity

$$
\varphi_{\ell+1 / 2, \lambda}-\varphi_{\ell, \lambda}=\varphi_{\ell+1, \lambda *} \hat{\mathbf{Q}}_{\ell}^{-1}-\varphi_{\ell, \lambda *} \hat{\mathbf{Q}}_{\ell}^{-1}=\varphi_{\ell, \lambda *} \hat{\mathbf{Q}}_{\ell}^{-1} \mathbf{S}_{\ell, \lambda}-\varphi_{\ell, \lambda *} \hat{\mathbf{Q}}_{\ell}^{-1}=\operatorname{ash}\left(\mathbf{S}_{\ell, \lambda}, e^{i \varphi_{\ell, \lambda}} \bar{\eta}_{\ell} \hat{\rho}_{\ell}^{-2},-1\right) .
$$

Here we used the definition of the angular shift with the fact that $\mathbf{S}_{\ell, \lambda}$ (and any affine transformation) will preserve $\infty \in \mathbb{H}$ which corresponds to -1 in $\mathbb{U}$. A similar identity can be proved for $\Delta_{1 / 2} \varphi_{\ell+1 / 2, \lambda}$.

The proof now follows exactly the same as in [13], it is a straightforward application of Lemma 12 using the estimates on $v_{\ell, \lambda}, \hat{v}_{\ell, \lambda}, V_{\ell}, \hat{V}_{\ell}$.

\subsection{The continuum limit}

In this section we will prove that $\varphi^{(n)}(t, \lambda)=\varphi_{\left\lfloor t n_{0}\right\rfloor, \lambda}$ converges to the solution of a one-parameter family of stochastic differential equations on $t \in[0,1)$. The main tool is the following proposition, proved in [13] (based on [11] and [4]).

Proposition 15. Fix $T>0$, and for each $n \geq 1$ consider a Markov chain $X_{\ell}^{n} \in \mathbb{R}^{d}$ with $\ell=1, \ldots,\lfloor n T\rfloor$. Let $Y_{\ell}^{n}(x)$ be distributed as the increment $X_{\ell+1}^{n}-x$ given $X_{\ell}^{n}=x$. We define

$$
b^{n}(t, x)=n E\left[Y_{\lfloor n t\rfloor}^{n}(x)\right], \quad a^{n}(t, x)=n E\left[Y_{\lfloor n t\rfloor}^{n}(x) Y_{\lfloor n t\rfloor}^{n}(x)^{\mathrm{T}}\right] .
$$


Suppose that as $n \rightarrow \infty$ we have

$$
\begin{aligned}
\left|a^{n}(t, x)-a^{n}(t, y)\right|+\left|b^{n}(t, x)-b^{n}(t, y)\right| & \leq c|x-y|+o(1) \\
\sup _{x, \ell} E\left[\left|Y_{\ell}^{n}(x)\right|^{3}\right] & \leq c n^{-3 / 2}
\end{aligned}
$$

and that there are functions $a, b$ from $\mathbb{R} \times[0, T]$ to $\mathbb{R}^{d^{2}}, \mathbb{R}^{d}$ respectively with bounded first and second derivatives so that

$$
\sup _{x \in \mathbb{R}^{d^{2}}, t}\left|\int_{0}^{t} a^{n}(s, x) d s-\int_{0}^{t} a(s, x) d s\right|+\sup _{x \in \mathbb{R}^{d}, t}\left|\int_{0}^{t} b^{n}(s, x) d s-\int_{0}^{t} b(s, x) d s\right| \rightarrow 0 .
$$

Assume also that the initial conditions converge weakly, $X_{0}^{n} \stackrel{d}{\Longrightarrow} X_{0}$.

Then $\left(X_{\lfloor n t\rfloor}^{n}, 0 \leq t \leq T\right)$ converges in law to the unique solution of the $S D E$

$$
d X=b d t+\sigma d B, \quad X(0)=X_{0}, \quad t \in[0, T],
$$

where $B$ is a d-dimensional standard Brownian motion and $\sigma: \mathbb{R}^{d} \times[0, T]$ is a square root of the matrix valued function a, i.e. $a(t, x)=\sigma(t, x) \sigma(t, x)^{T}$.

We will apply this proposition to $\varphi_{\ell, \lambda}$ with $\ell \leq n_{0}(1-\varepsilon)$ and $\ell \in \mathbb{Z} / 2$, so the single steps of the proposition correspond to half steps in our setup.

The following lemma shows that the oscillatory terms in the estimates of Proposition 14 average out in the 'long run'. Its proof relies on Proposition 14 and Lemma 26 of the Appendix.

Lemma 16. Let $|\lambda|,\left|\lambda^{\prime}\right| \leq \bar{\lambda}$ and $\varepsilon>0$. Then for any $\ell_{1} \leq n_{0}(1-\varepsilon), \ell_{1} \in \mathbb{Z}$

$$
\begin{aligned}
& \frac{1}{n_{0}} \sum_{0 \leq \ell<\ell_{1}}^{\sim} \mathbb{E}\left[\Delta_{1 / 2} \varphi_{\ell, \lambda} \mid \varphi_{\ell, \lambda}=x\right]=\frac{1}{n_{0}} \sum_{\ell=0}^{\ell_{1}-1} b_{\lambda}(t)+\mathscr{O}\left(n_{0}^{-1 / 2}+n_{1}^{1 / 2} n_{0}^{-3 / 2}\right) \\
& \frac{1}{n_{0}} \sum_{0 \leq \ell<\ell_{1}}^{\sim} \mathbb{E}\left[\Delta_{1 / 2} \varphi_{\ell, \lambda} \Delta_{1 / 2} \varphi_{\ell, \lambda^{\prime}} \mid \varphi_{\ell, \lambda}=x, \varphi_{\ell, \lambda^{\prime}}=y\right]=\frac{1}{n_{0}} \sum_{\ell=0}^{\ell_{1}-1} a(t, x, y)+\mathscr{O}\left(n_{0}^{-1 / 2}+n_{1}^{1 / 2} n_{0}^{-3 / 2}\right)
\end{aligned}
$$

where $t=\ell / n_{0}$, the functions $b_{\lambda}$, a are defined as

$$
b_{\lambda}=\frac{\lambda}{2 \hat{s}}+\frac{\Re \hat{\rho}}{2 p \hat{s}}+\frac{\mathfrak{I}\left(\hat{\rho}^{2}+\rho^{2}\right)}{2 \beta \hat{s}^{2}}-\frac{\Re \frac{d}{d t} \rho}{\mathfrak{I} \rho}, \quad a=\frac{2}{\beta \hat{s}^{2}} \Re\left[e^{i(x-y)}\right]+\frac{2+\Re\left(\hat{\rho}^{2}+\rho^{2}\right)}{\beta \hat{s}^{2}},
$$

and the implicit constants in $\mathscr{O}$ depend only on $\varepsilon, \beta, \bar{\lambda}$. The indices in the summation $\tilde{\sum}$ run through half integers.

Proof of Lemma 16. We will only prove the first statement, the second one being similar. Note that $b_{\lambda}(t)=b_{\lambda}^{(1)}(t)+b_{\lambda}^{(2)}(t)$.

Summing the first and third estimates in Proposition 14 we get (51) with an error term

$$
\frac{1}{n_{0}} \sum_{\ell=0}^{\ell_{1}-1} \Re\left(e_{1, \ell} \eta_{\ell}\right)+\frac{1}{n_{0}} \sum_{\ell=0}^{\ell_{1}-1} \Re\left(e_{2, \ell} \eta_{\ell}^{2}\right)+\mathscr{O}\left(n_{0}^{-1 / 2}\right)
$$


where the first two terms will be denoted $\zeta_{1}, \zeta_{2}$. Here

$$
e_{1, \ell}=\left(\left(-v_{\lambda}-i q^{(1)} / 2\right) \hat{\rho}_{\ell}^{2}+\left(-\hat{v}_{\lambda}-i q^{(2)} / 2\right)\right) e^{-i x}, \quad e_{2, \ell}=i\left(\hat{\rho}_{\ell}^{-4} q^{(1)}+q^{(2)}\right) e^{-2 i x} / 4
$$

where for this proof $c$ denotes varying constants depending on $\varepsilon$. Using the fact that $v_{\lambda}, \hat{v}_{\lambda}, q^{(1)}, q^{(2)}$ and their first derivatives are continuous on $[0,1-\varepsilon]$ we get

$$
\left|e_{i, \ell}\right|<c, \quad\left|e_{i, \ell}-e_{i, \ell+1}\right|<c n_{0}^{-1} \text {. }
$$

Applying Lemma 26 of the Appendix to the first sum in (53):

$$
\left|\zeta_{1}\right| \leq \frac{1}{n_{0}}\left|e_{1, \ell_{1}}\right|\left|F_{1, \ell_{1}}^{(1)}\right|+\frac{1}{n_{0}} \sum_{\ell=1}^{\ell_{1}-1}\left|e_{1, \ell}-e_{1, \ell+1}\right|\left|F_{1, \ell}^{(1)}\right| .
$$

Since $\ell_{1} \leq n_{0}(1-\varepsilon)$ we have $\left|F_{1, \ell}^{(1)}\right| \leq c\left(1+n_{1}^{1 / 2} k^{-1 / 2}\right) \leq c\left(n_{1}^{1 / 2} n_{0}^{-1 / 2}+1\right)$ and

$$
\left|\zeta_{1}\right| \leq c\left(n_{0}^{-3 / 2} n_{1}^{1 / 2}+n_{0}^{-1}\right)
$$

(Recall that $k=n_{0}-\ell$.) For the estimate of $\zeta_{2}$ we first note that

$$
\left|e_{2, \ell}\right|=\frac{1}{2 \beta} \frac{n_{0}}{k}\left|\hat{\rho}_{\ell}^{-2}+\rho_{\ell}^{2}\right|=\frac{1}{2 \beta} \frac{n_{0}}{k}\left|\hat{\rho}_{\ell}^{2} \rho_{\ell}^{2}+1\right| .
$$

We will use Lemma 26 if $\left|\hat{\rho}_{\ell}^{2} \rho_{\ell}^{2}+1\right|$ is 'big', and a direct bound with (55) if it is small. To be more precise: we divide the sum into three pieces, we cut it at indices $\ell_{1}^{*}$ and $\ell_{2}^{*}$ so that

$$
\left|\hat{\rho}_{\ell}^{2} \rho_{\ell}^{2}+1\right| \leq n_{0}^{-1 / 2} \quad \text { if } k \in\left[k_{2}^{*}, k_{1}^{*}\right] \quad \text { and }\left|\hat{\rho}_{\ell}^{2} \rho_{\ell}^{2}+1\right| \geq n_{0}^{-1 / 2} \text { otherwise. }
$$

Note that one or two of the resulting partial sums may be empty. We can always find such indices because $\arg \hat{\rho}_{\ell}^{2} \rho_{\ell}^{2}$ is monotone if $\mu_{n} \geq \sqrt{m-n}$ and if $\mu_{n}<\sqrt{m-n}$ then $\arg \hat{\rho}_{\ell}^{2} \rho_{\ell}^{2}$ decreases if $k>\sqrt{m_{1} n_{1}}$ then it increases. (See the proof of Lemma 26.)

We denote the three pieces by $\zeta_{2, i}, i=1,2,3$ and bound them separately. Since $k \geq \varepsilon n_{0}$, Lemma 26 gives

$$
\left|\zeta_{2,1}\right| \leq c\left(n_{1}^{1 / 2} n_{0}^{-3 / 2}+n_{0}^{-1 / 2}\right) .
$$

The term $\left|\zeta_{2,3}\right|$ can be bounded exactly the same way, so we only need to deal with $\zeta_{2,2}$. Here we use (55) to get a direct estimate:

$$
\left|\zeta_{2,2}\right| \leq \frac{1}{2 \beta} \sum_{k \in\left[k_{2}^{*}, k_{1}^{*}\right] \cap\left[\varepsilon n_{0}, n_{0}\right]} \frac{1}{k}\left|\hat{\rho}_{\ell}^{-2}+\rho_{\ell}^{2}\right| \leq c n_{0}^{-1 / 2} .
$$

Collecting all our estimates the statement follows.

Now we have the ingredients to prove the continuum limit. 
Proposition 17. Assume that $m / n_{0} \rightarrow \kappa \in[1, \infty], n / n_{0} \rightarrow v \in[1, \infty]$ and that eventually $\mu_{n}>\sqrt{m-n}$ or $\mu_{n} \leq \sqrt{m-n}$. Then the continuous functions $p^{(n)}(t)^{-1}, \rho^{(n)}(t), \hat{\rho}^{(n)}(t)$ converge to following limits on $[0,1)$ :

$$
p^{-1}(t)=(\kappa-t)^{-1 / 2}, \quad \rho(t)= \pm \sqrt{\frac{v-1}{v-t}}+i \sqrt{\frac{1-t}{v-t}}, \quad \hat{\rho}(t)=\sqrt{\frac{\kappa-1}{\kappa-t}}+i \sqrt{\frac{1-t}{\kappa-t}}
$$

where the sign in $\Re \rho$ depends on the (eventual) sign of $\mu_{n}-\sqrt{m-n}$. If $\kappa=\infty$ then $p^{-1}(t)=0$, $\hat{\rho}(t)=1$ and if $v=\infty$ then $\rho(t)= \pm 1$.

Let $B$ and $W$ be independent real and complex standard Brownian motions, and for each $\lambda \in \mathbb{R}$ consider the strong solution of

$$
\begin{aligned}
d \varphi_{\lambda} & =\left[\frac{\lambda}{2 \hat{s}}-\frac{\Re \rho^{\prime}}{\mathfrak{I} \rho}+\frac{\mathfrak{I}\left(\rho^{2}+\hat{\rho}^{2}\right)}{2 \beta \hat{s}^{2}}+\frac{\Re \hat{\rho}}{2 p \hat{s}}\right] d t+\frac{\sqrt{2} \Re\left(e^{-i \varphi_{\lambda}} d W\right)}{\sqrt{\beta} \hat{s}}+\frac{\sqrt{2+\Re\left(\rho^{2}+\hat{\rho}^{2}\right)}}{\sqrt{\beta} \hat{s}} d B, \\
\varphi_{\lambda}(0) & =\pi .
\end{aligned}
$$

Then we have

$$
\varphi_{\lambda,\left\lfloor n_{0} t\right\rfloor} \stackrel{d}{\Longrightarrow} \varphi_{\lambda}(t), \quad \text { as } n \rightarrow \infty,
$$

where the convergence is in the sense of finite dimensional distributions for $\lambda$ and in path-space $D[0,1)$ for $t$.

Proof. The proof is very similar to the proof of Theorem 25 in [13]. One needs to check that for any fixed vector $\left(\lambda_{1}, \ldots, \lambda_{d}\right)$ the Markov chain $\left(\varphi_{\ell, \lambda_{i}}, 1 \leq i \leq d\right), \ell \leq\left\lfloor(1-\varepsilon) n_{0}\right\rfloor, \ell \in \mathbb{Z} / 2$ satisfies the conditions of Proposition 15 and to identify the variance matrix of the limiting diffusion. Note that because our Markov chain lives on the half integers one needs to slightly rephrase the proposition, but this is straightforward.

The Lipshitz condition (48) and the moment condition (49) are easy to check from Proposition 14. The averaging condition (50) is satisfied because of Lemma 16, using the fact that because of the conditions of the proposition, the functions $b^{\lambda}(t), a(t, x, y)$ converge. This proves that the rescaled version of $\left(\varphi_{\ell, \lambda_{j}}, 1 \leq j \leq d\right)$ converges in distribution to an SDE in $\mathbb{R}^{d}$ where the drift term is given by the limit of $\left(b_{j}^{\lambda}, j=1 \ldots d\right)$ and the diffusion matrix is given by $a(t, x)_{j, k}=\frac{2}{\beta \hat{s}^{2}} \Re\left[e^{i\left(x_{k}-x_{j}\right)}\right]+$ $\frac{2+\Re\left(\hat{\rho}^{2}+\rho^{2}\right)}{\beta \hat{s}^{2}}$.

The only step left is to verify that the limiting SDE can be rewritten in the form (57). This follows easily using the fact that if $Z$ is a complex Gaussian with i.i.d. standard real and imaginary parts and $\omega_{1}, \omega_{2} \in \mathbb{C}$ then

$$
\mathbb{E} \Re\left(\omega_{1} Z\right) \Re\left(\omega_{2} Z\right)=\Re\left(\bar{\omega}_{1} \omega_{2}\right) .
$$

The following corollary describes the scaling limit of the relative phase function $\alpha_{\ell, \lambda}$.

Corollary 18. Let $Z$ be a complex Brownian motion with i.i.d. standard real and imaginary parts and consider the strong solution $\alpha_{\lambda}(t)$ of the SDE system $\{17)$. Then $\alpha_{\left\lfloor n_{0} t\right\rfloor, \lambda} \stackrel{d}{\Longrightarrow} \alpha_{\lambda}(t)$ as $n \rightarrow \infty$ where the convergence is in the sense of finite dimensional distributions for $\lambda$ and in path-space $D[0,1)$ for $t$. 
Proof. We just need to show that for any subsequence of $n$ we can choose a further subsequence so that the convergence holds. By choosing an appropriate subsequence we can assume that $m / n_{0}, n / n_{0}$ both converge and that $\mu_{n}-\sqrt{m-n}$ is always positive or nonnegative. Then the conditions of Proposition 17 are satisfied and $\alpha_{\lambda}=\varphi_{\lambda}-\varphi_{0}$ will satisfy the SDE (17) with a complex Brownian motion $Z_{t}:=\int_{0}^{t} e^{i \varphi_{0}(t)} d W_{t}$. From this the statement of the corollary follows.

\section{Middle stretch}

In this section we will study the behavior of $\alpha_{\ell, \lambda}$ and $\varphi_{\ell, \lambda}$ in the interval $\left[\left\lfloor(1-\varepsilon) n_{0}\right\rfloor, n_{2}\right]$ with $n_{2}=$ $\left\lfloor n_{0}-\mathscr{K}\left(n_{1}^{1 / 3} \vee 1\right)\right\rfloor$. The constant $\mathscr{K}$ will eventually go to $\infty$, so we can assume that $\mathscr{K}>C_{0}>0$ with $C_{0}$ large enough.

\subsection{The relative phase function}

The objective of this subsection is to show that the relative phase function $\alpha_{\ell, \lambda}$ does not change much in the middle stretch.

Proposition 19. There exists a constant $c=c(\bar{\lambda}, \beta)$ so that with $y=n_{0}^{-1 / 2}\left(n_{1}^{1 / 6} \vee \log n_{0}\right)$ we have

$$
\mathbb{E}\left[\left|\alpha_{\ell_{2}, \lambda}-\alpha_{\ell_{1}, \lambda}\right| \wedge 1 \mid \mathscr{F}_{\ell_{1}}\right] \leq c\left(d\left(\alpha_{\ell_{1}, \lambda}, 2 \pi \mathbb{Z}\right)+\sqrt{\epsilon}+y+\mathscr{K}^{-1}\right)
$$

for all $\mathscr{K}>0, \epsilon \in(0,1), \lambda \leq|\bar{\lambda}|, n_{0}(1-\varepsilon) \leq l_{1} \leq l_{2} \leq n_{2}, \ell \in \mathbb{Z}$.

Because of the moment bounds (30) we may assume that

$$
\left|X_{\ell}\right|,\left|Y_{\ell}\right| \leq \frac{1}{10} \sqrt{n_{0}} \hat{s}\left(\ell / n_{0}\right), \quad \text { for } \ell \leq n_{2} .
$$

Indeed, the probability that (59) does not hold is at most $c\left(n_{0}-n_{2}\right)^{-1} \leq c \mathscr{K}^{-1}$ which can be absorbed in the error term of (58).

We first provide the one-step estimates for the evolution of the relative phase function.

Proposition 20. There exists $c=c(\beta, \bar{\lambda})$ so that for every $\ell \leq n_{2}$ and $|\lambda|<\bar{\lambda}$ we have the following estimates

$$
\begin{aligned}
& \mathbb{E}\left(\Delta \alpha_{\ell, \lambda} \mid \mathscr{F}_{\ell}\right)=-\frac{1}{n_{0}} \Re\left\{\eta_{\ell}\left(e^{-i \varphi_{\ell, \lambda}}-e^{-i \varphi_{\ell, 0}}\right)\left[\hat{\rho}_{\ell}^{-2}\left(v_{\lambda}+i q^{(1)} / 2\right)+\left(\hat{v}_{\lambda}+i q^{(2)} / 2\right)\right]\right\} \\
& \quad-\frac{1}{n_{0}} \Re\left\{i \eta_{\ell}^{2} / 4\left(e^{-2 i \varphi_{\ell, \lambda}}-e^{-2 i \varphi_{\ell, 0}}\right)\left[\hat{\rho}_{\ell}^{-4} q^{(1)}+q^{(2)}\right]\right\}+\mathscr{O}\left(\hat{\alpha}_{\ell, \lambda} k^{-3 / 2}+k^{-1 / 2} n_{0}^{-1 / 2}\right) \\
& \quad=\mathscr{O}\left(\hat{\alpha}_{\ell, \lambda} k^{-1}+k^{-1 / 2} n_{0}^{-1 / 2}\right) \\
& \mathbb{E}\left(\Delta \alpha_{\ell, \lambda}^{2} \mid \mathscr{F}_{\ell}\right)=\mathscr{O}\left(\hat{\alpha}_{\ell, \lambda} k^{-1}+k^{-1} n_{0}^{-1}\right) \\
& \mathbb{E}\left(\left|\Delta \alpha_{\ell, \lambda} \Delta \varphi_{\ell, \lambda}\right| \mid \mathscr{F}_{\ell}\right)=\mathscr{O}\left(\hat{\alpha}_{\ell, \lambda} k^{-1}\right)
\end{aligned}
$$

where $\hat{\alpha}_{\ell, \lambda}$ denotes the distance between $\alpha_{\ell, \lambda}$ and $2 \pi$. 
Proof. We first prove estimates on $\Delta_{1 / 2} \alpha_{\ell, \lambda}$ and $\Delta_{1 / 2} \alpha_{\ell+1 / 2, \lambda}$. In order to do this, we break up the evolution of $\varphi_{\ell, \lambda}$ into even smaller pieces:

$$
\varphi_{\ell, \lambda} \stackrel{\mathbf{L}_{\ell}^{\hat{\mathbf{T}}_{\ell}} \hat{\mathbf{Q}}_{\ell}}{\longrightarrow} \varphi_{\ell+1 / 4, \lambda} \stackrel{\mathbf{s}_{\ell, 0}^{\hat{Q}_{\ell}}}{\longrightarrow} \varphi_{\ell+1 / 2, \lambda} \stackrel{\hat{\mathbf{L}}_{\ell} \mathbf{T}_{\ell} \mathbf{Q}_{\ell}}{\longrightarrow} \varphi_{\ell+3 / 4, \lambda} \stackrel{\hat{\mathbf{s}}_{\ell, 0}^{\mathbf{Q}_{\ell}}}{\longrightarrow} \varphi_{\ell+1, \lambda}
$$

where $\varphi_{\ell+1 / 4, \lambda}$ and $\varphi_{\ell+3 / 4, \lambda}$ are defined accordingly. We also define the relative phase functions for the intermediate steps in the natural way.

By choosing $c(\beta, \bar{\lambda})$ large enough we can assume $\frac{\bar{\lambda}}{4 \sqrt{n_{0} k}} \leq \frac{1}{10}$ for $\ell \leq n_{2} \leq n-\mathscr{K}$. Using this with the cutoff (59) the random variables $Z_{\ell, \lambda}, \hat{Z}_{\ell, \lambda}$ defined in (46) are both less than $1 / 3$ in absolute value. This means that we are allowed to use Lemma 12 in the general case for each operator appearing in (63) (i.e. the condition $|z| \leq 1 / 3$ is always satisfied). From this point the proof is similar to the proof of Proposition 29 in [13]. We first write

$$
\begin{gathered}
\Delta_{1 / 2} \alpha_{\ell, \lambda}=\operatorname{ash}\left(\mathbf{L}_{\ell}^{\hat{\mathbf{T}}_{\ell}},-1, e^{i \varphi_{\ell, \lambda}} \bar{\eta}_{\ell} \hat{\rho}_{\ell}^{-2}\right)+\operatorname{ash}\left(\mathbf{S}_{\ell, 0}^{\hat{\mathbf{Q}}_{\ell}}, e^{i \varphi_{\ell+1 / 4, \lambda}} \bar{\eta}_{\ell} \hat{\rho}_{\ell}^{-2}, e^{i \varphi_{\ell, \lambda}} \bar{\eta}_{\ell} \hat{\rho}_{\ell}^{-2}\right) \\
+\operatorname{ash}\left(\mathbf{S}_{\ell, 0}^{\hat{\mathbf{Q}}_{\ell}}, e^{i \varphi_{\ell, \lambda}} \bar{\eta}_{\ell} \hat{\rho}_{\ell}^{-2}, e^{i \varphi_{\ell, 0}} \bar{\eta}_{\ell} \hat{\rho}_{\ell}^{-2}\right) .
\end{gathered}
$$

Using Lemma 12 one can show that the first two terms in 64 are of $\mathscr{O}\left(n_{0}^{-1 / 2} k^{-1 / 2}\right)$. Using Lemma 12 again for the third term together with

$$
\left|e^{i \varphi_{\ell, \lambda}}-e^{i \varphi_{\ell, 0}}\right|=\left|e^{i \alpha_{\ell, \lambda}}-1\right| \leq \hat{\alpha}_{\ell, \lambda}, \quad\left|e^{i 2 \varphi_{\ell, \lambda}}-e^{i 2 \varphi_{\ell, 0}}\right| \leq 2 \hat{\alpha}_{\ell, \lambda}
$$

we get the analogue of 60 for $\Delta_{1 / 2} \alpha_{\ell, \lambda}$ :

$$
\begin{gathered}
\mathbb{E}\left(\Delta_{1 / 2} \alpha_{\ell, \lambda} \mid \mathscr{F}_{\ell}\right)=-\frac{1}{n_{0}} \Re\left\{\eta_{\ell}\left(e^{-i \varphi_{\ell, \lambda}}-e^{-i \varphi_{\ell, 0}}\right)\left[\hat{\rho}_{\ell}^{-2}\left(v_{\lambda}+i q^{(1)} / 2\right)\right]\right\} \\
-\frac{1}{n_{0}} \Re\left\{i \eta_{\ell}^{2} / 4\left(e^{-2 i \varphi_{\ell, \lambda}}-e^{-2 i \varphi_{\ell, 0}}\right)\left[\hat{\rho}_{\ell}^{-4} q^{(1)}\right]\right\}+\mathscr{O}\left(\hat{\alpha}_{\ell, \lambda} k^{-3 / 2}+k^{-1 / 2} n_{0}^{-1 / 2}\right) \\
=\mathscr{O}\left(\hat{\alpha}_{\ell, \lambda} k^{-1}+k^{-1 / 2} n_{0}^{-1 / 2}\right)
\end{gathered}
$$

We can prove the analogues of (61) and (62) and similar bounds for $\Delta_{1 / 2} \alpha_{\ell+1 / 2, \lambda}$ the same way. We can also prove

$$
\mathbb{E}\left(\Delta_{1 / 2} e^{i \varphi_{\ell, \lambda}}-\Delta_{1 / 2} e^{i \varphi_{\ell, 0}} \mid \mathscr{F}_{\ell}\right) \leq c k^{-1} \hat{\alpha}_{\ell}+c n_{0}^{-1 / 2} k^{-1 / 2}
$$

this is the analogue of Lemma 32 from [13] and it can be proved exactly the same way.

To get (60) we write

$$
\begin{aligned}
& \mathbb{E}\left[\Delta \alpha_{\ell, \lambda} \mid \varphi_{\ell, 0}=x, \varphi_{\ell, \lambda}=y\right] \\
& =\mathbb{E}\left[\Delta_{1 / 2} \alpha_{\ell, \lambda} \mid \varphi_{\ell, 0}=x, \varphi_{\ell, \lambda}=y\right]+\mathbb{E}\left[E\left[\Delta_{1 / 2} \alpha_{\ell+1 / 2, \lambda} \mid \mathscr{F}_{\ell+1 / 2}\right] \mid \varphi_{\ell, 0}=x, \varphi_{\ell, \lambda}=y\right], \\
& =\mathbb{E}\left[\Delta_{1 / 2} \alpha_{\ell, \lambda} \mid \varphi_{\ell, 0}=x, \varphi_{\ell, \lambda}=y\right]+\mathbb{E}\left[\Delta_{1 / 2} \alpha_{\ell+1 / 2, \lambda} \mid \varphi_{\ell+1 / 2,0}=x, \varphi_{\ell+1 / 2, \lambda}=y\right] \\
& +\mathscr{O}\left(\hat{\alpha}_{\ell, \lambda} k^{-2}+k^{-3 / 2} n_{0}^{-1 / 2}\right)
\end{aligned}
$$

where the last line follows from (66) and the just proved half step estimates. Now applying (65) and the corresponding estimate for $\Delta_{1 / 2} \alpha_{\ell+1 / 2, \lambda}$ we get (60). The other two estimates follow similarly. 
The next lemma provides a Gronwall-type estimate for the relative phase function. This will be the main ingredient in the proof of Proposition 19. The proof is based on the single step estimates of Proposition 20 and the oscillation estimates of Lemma 26, the latter will be proved in the Appendix.

Lemma 21. There exist constants $c_{0}, c_{1}, c_{2}$ depending on $\bar{\lambda}, \beta$ and a finite set $J$ depending on $n, n_{1}, m_{1}$ so that with $y=n_{0}^{-1 / 2}\left(n_{1}^{1 / 6} \vee \log n_{0}\right)$ we have

$$
\begin{gathered}
\left|\mathbb{E}\left(\alpha_{\ell_{2}, \lambda}-\alpha_{\ell_{1}, \lambda} \mid \mathscr{F}_{\ell_{1}}\right)\right| \leq c_{1}(y+\sqrt{\epsilon})+\mathbb{E}\left(\hat{\alpha}_{\ell_{2}-1} \mid \mathscr{F}_{\ell_{1}}\right) / 2+\sum_{\ell=\ell_{1}}^{\ell_{2}-2} b_{\ell} \mathbb{E}\left(\hat{\alpha}_{\ell} \mid \mathscr{F}_{\ell}\right) \\
0 \leq b_{\ell} \leq c_{0}\left(n_{1}^{1 / 2} k^{-5 / 2}+k^{-3 / 2}+\left(n_{1}^{1 / 3} \vee 1\right) k^{-2}+1_{(\ell \in J)}\right)
\end{gathered}
$$

if $\mathscr{K}>c_{2},|\lambda| \leq \bar{\lambda}$ and $n_{0}(1-\varepsilon) \leq \ell_{1} \leq \ell_{2} \leq n_{2}$.

Proof. Recall that $k=n_{0}-\ell, k_{i}=n_{0}-\ell_{i}$. We denote $x_{\ell}=\mathbb{E}\left[\hat{\alpha}_{\ell} \mid \mathscr{F}_{\ell_{1}}\right]$ and set

$$
\begin{aligned}
e_{1, \ell} & =\frac{1}{n_{0}}\left(e^{-i \varphi_{\ell, \lambda}}-e^{-i \varphi_{\ell, 0}}\right)\left[\left(-v_{\lambda}-i q^{(1)} / 2\right) \hat{\rho}_{\ell}^{-2}+\left(-\hat{v}_{0}-i q^{(2)} / 2\right)\right], \\
e_{2, \ell} & =-\frac{i}{n_{0}}\left(e^{-2 i \varphi_{\ell, \lambda}}-e^{-2 i \varphi_{\ell, 0}}\right)\left[\hat{\rho}_{\ell}^{-4} q^{(1)}+q^{(2)}\right] / 4 .
\end{aligned}
$$

From Proposition 20 we can write

$$
\left|\mathbb{E}\left[\alpha_{2}-\alpha_{1} \mid \mathscr{F}_{\ell_{1}}\right]\right| \leq\left|\sum_{\ell=\ell_{1}}^{\ell_{2}-1} \Re\left(\eta_{\ell} e_{1, \ell}\right)\right|+\left|\sum_{\ell=\ell_{1}}^{\ell_{2}-1} \Re\left(\eta_{\ell}^{2} e_{2, \ell}\right)\right|+c \sum_{\ell=\ell_{1}}^{\ell_{2}-1} x_{\ell} k^{-3 / 2}+c \sum_{\ell=\ell_{1}}^{\ell_{2}-1} k^{-1 / 2} n_{0}^{-1 / 2}
$$

whose terms we denote $\zeta_{1}, \zeta_{2}, \zeta_{3}$ and $\zeta_{4}$ respectively. Clearly, $\zeta_{3}$ is of the right form and

$$
\left|\zeta_{4}\right| \leq \sum_{k=1}^{n_{0} \varepsilon} k^{-1 / 2} n_{0}^{-1 / 2} \leq c \sqrt{\varepsilon}
$$

so we only need to bound the first two terms.

We will use

$$
\mathbb{E}\left(\Delta e^{i \varphi_{\ell, \lambda}}-\Delta e^{i \varphi_{\ell, 0}} \mid \mathscr{F}_{\ell}\right) \leq c k^{-1} x_{\ell}+c n_{0}^{-1 / 2} k^{-1 / 2}
$$

which is the 'one-step' version of (66) and can be proved the same way as Lemma 32 in [13]. From this we get the estimates

$$
\left|e_{i, \ell}\right| \leq c x_{\ell} / k, \quad\left|\Delta e_{i, \ell}\right| \leq c k^{-2} x_{\ell}+c n_{0}^{-1 / 2} k^{-3 / 2} .
$$

Then by Lemma 26 we have

$$
\left|\zeta_{1}\right| \leq C x_{\ell_{2}-1} k_{2}^{-1}\left|F_{\ell_{1}, \ell_{2}-1}^{(1)}\right|+C \sum_{\ell=\ell_{1}}^{\ell_{2}-2}\left|F_{\ell_{1}, \ell}^{(1)}\right|\left(x_{\ell} k^{-2}+n_{0}^{-1 / 2} k^{-3 / 2}\right)
$$

with $F_{\ell_{1}, \ell} \leq C\left(n_{1}^{1 / 2} k^{-1 / 2}+1\right)$. Collecting the estimates and using $k_{2} \geq \mathscr{K}\left(n_{1}^{1 / 3} \vee 1\right)$ we get

$$
\left|\zeta_{1}\right| \leq c \mathscr{K}^{-1} x_{\ell_{2}-1}+c n_{0}^{-1 / 2}\left(n_{1}^{1 / 6} \vee 1\right)+\sum_{\ell=\ell_{1}}^{\ell_{2}-2} x_{\ell}\left(n_{1}^{1 / 2} k^{-5 / 2}+k^{-2}\right) \text {. }
$$


In order to bound $\zeta_{2}$ we use a similar strategy to the one applied in the proof of Lemma 16 . We divide the index set $\left[\ell_{1}, \ell_{2}\right]$ into finitely many intervals $I_{1}, I_{2}, \ldots, I_{a}$ so that for each $1 \leq j \leq a$ one of the following three statements holds:

$$
\begin{aligned}
& \text { for each } \ell \in I_{j} \text { we have } k \geq \sqrt{n_{1} m_{1}} \text { and }\left|\hat{\rho}_{\ell}^{2} \rho_{\ell}^{2}+1\right| \geq k^{-1 / 2} \text {, } \\
& \text { for each } \ell \in I_{j} \text { we have } k \leq \sqrt{n_{1} m_{1}} \text { and }\left|\hat{\rho}_{\ell}^{2} \rho_{\ell}^{2}+1\right| \geq k^{-1 / 2} \text {, } \\
& \text { for each } \ell \in I_{j} \text { we have }\left|\hat{\rho}_{\ell}^{2} \rho_{\ell}^{2}+1\right| \leq k^{-1 / 2} \text {. }
\end{aligned}
$$

It is clear that if we divide $\left[\ell_{1}, \ell_{2}\right]$ into parts at the $\sqrt{n_{1} m_{1}}$ and the solutions of $\left|\hat{\rho}_{\ell}^{2} \rho_{\ell}^{2}+1\right|=k^{-1 / 2}$ then the resulting partition will satisfy the previous conditions. Since $\left|\hat{\rho}_{\ell}^{2} \rho_{\ell}^{2}+1\right|=k^{-1 / 2}$ has at most three roots (it is a cubic equation, see the proof of Lemma 26 for details) we can always get a suitable partition with at most five intervals. Moreover the endpoints of these intervals (apart from $\ell_{1}$ and $\ell_{2}$ ) will be the elements of a set of size at most four with elements only depending on $n, m_{1}, n_{1}$.

We will estimate the sums corresponding to the various intervals $I_{j}$ separately. If $I_{j}$ satisfies condition (69) then we use

$$
\left|e_{2, \ell}\right|=\frac{1}{2 \beta}\left|e^{-2 i x}-e^{-2 i y}\right| \frac{1}{k}\left|\hat{\rho}_{\ell}^{2} \rho_{\ell}^{2}+1\right| \leq c k^{-3 / 2} x_{\ell}
$$

to get

$$
\left|\sum_{\ell \in I_{j}} \Re\left(\eta_{\ell}^{2} e_{2, \ell}\right)\right| \leq c \sum_{\ell \in I_{j}} k^{-3 / 2} x_{\ell}
$$

If $I_{j}=\left[\ell_{1}^{*}, \ell_{2}^{*}\right]$ satisfies condition (67) then we use Lemma 26 to get

$$
\left|\sum_{\ell \in I_{j}} \Re\left(\eta_{\ell}^{2} e_{2, \ell}\right)\right| \leq c x_{\ell_{1}^{*}}\left(k_{1}^{*}\right)^{-1}\left|F_{\ell_{1}^{*}, \ell_{2}^{*}}^{(2)}\right|+c \sum_{\ell=\ell_{1}^{*}}^{\ell_{2}^{*}-1}\left|F_{\ell+1, \ell_{2}^{*}}^{(2)}\right|\left(x_{\ell} k^{-2}+n_{0}^{-1 / 2} k^{-3 / 2}\right)
$$

where we have

$$
\left|F_{\ell+1, \ell_{2}^{*}}^{(2)}\right| \leq\left|F_{\ell, \ell_{2}^{*}}^{(2)}\right|+1 \leq c\left(k^{1 / 2}+n_{1}^{1 / 2}\left(k_{2}^{*}\right)^{-1 / 2}+1\right) .
$$

We can bound the first term as

$$
x_{\ell_{1}^{*}}\left(k_{1}^{*}\right)^{-1}\left(\left(k_{1}^{*}\right)^{1 / 2}+n_{1}^{1 / 2}\left(k_{2}^{*}\right)^{-1 / 2}+1\right) \leq c \mathscr{K}^{-1 / 2} x_{\ell_{1}^{*}}
$$

using $k \geq\left(n_{1}^{1 / 3} \vee 1\right)$. For the general term in the sum we get

$$
\begin{aligned}
c\left(k^{1 / 2}+n_{1}^{1 / 2}\right. & \left.\left(k_{2}^{*}\right)^{-1 / 2}+1\right)\left(x_{\ell} k^{-2}+n_{0}^{-1 / 2} k^{-3 / 2}\right) \\
& \leq c x_{\ell}\left(k^{-3 / 2}+\left(n_{1}^{1 / 3} \vee 1\right) k^{-2}\right)+c\left(n_{0}^{-1 / 2} k^{-1}+\left(n_{1}^{1 / 3} \vee 1\right) n_{0}^{-1 / 2} k^{-3 / 2}\right)
\end{aligned}
$$

Note that the sum of the error terms $(71)$ is

$$
c \sum_{\ell=\ell_{1}^{*}}^{\ell_{2}^{*}-1}\left(n_{0}^{-1 / 2} k^{-1}+\left(n_{1}^{1 / 3} \vee 1\right) n_{0}^{-1 / 2} k^{-3 / 2}\right) \leq c\left(n_{0}^{-1 / 2} \log n_{0}+\left(n_{1}^{1 / 6} \vee 1\right) n_{0}^{-1 / 2}\right)
$$


where we used $\left(n_{1}^{1 / 3} \vee 1\right) \leq k \leq n_{0}$. Putting our estimates together:

$$
\begin{aligned}
\left|\sum_{\ell \in I_{j}} \Re\left(\eta_{\ell}^{2} e_{2, \ell}\right)\right| \leq c \mathscr{K}^{-1 / 2} x_{\ell_{1}^{*}} & +c \sum_{\ell=\ell_{1}}^{\ell_{1}^{*}-1} x_{\ell}\left(k^{-3 / 2}+\left(n_{1}^{1 / 3} \vee 1\right) k^{-2}\right) \\
& +c\left(n_{0}^{-1 / 2} \log n_{0}+\left(n_{1}^{1 / 6} \vee 1\right) n_{0}^{-1 / 2}\right)
\end{aligned}
$$

The only case left is when $I_{j}=\left[\ell_{1}^{*}, \ell_{2}^{*}\right]$ satisfies condition (68). If $\mu_{n} \geq \sqrt{m-n}$ then we have the same estimate for $F_{\ell, \ell_{2}^{*}}^{(2)}$ as in 70 so we get exactly the same bound as in 72 . If we have $\mu_{n}<\sqrt{m-n}$ then we use (80) of Lemma with the bound

$$
\left|F_{\ell_{1}^{*}, \ell}^{(2)}\right| \leq c\left(k^{1 / 2}+n_{1}^{1 / 2}\left(k_{1}^{*}\right)^{-1 / 2}+1\right) .
$$

Copying the previous arguments we get

$$
\begin{array}{r}
\left|\sum_{\ell \in I_{j}} \Re\left(\eta_{\ell}^{2} e_{2, \ell}\right)\right| \leq c \mathscr{K}^{-1 / 2} x_{\ell_{2}^{*}-1}+c \sum_{\ell=\ell_{1}^{*}}^{\ell_{2}^{*}-1} x_{\ell}\left(k^{-3 / 2}+\left(n_{1}^{1 / 3} \vee 1\right) k^{-2}\right) \\
+c\left(n_{0}^{-1 / 2} \log n_{0}+\left(n_{1}^{1 / 6} \vee 1\right) n_{0}^{-1 / 2}\right)
\end{array}
$$

Collecting our estimates, noting that $\ell_{2}^{*}-1$ is the endpoint of one of the intervals $I_{j}$ and letting $\mathscr{K}$ be large enough we get the statement of the lemma.

The proof of Proposition 19 relies on the single step estimates of Proposition 20 and the following Gronwall-type lemma which was proved in [13].

Lemma 22. Suppose that for positive numbers $x_{\ell}, b_{\ell}, c$, integers $\ell_{1}<\ell \leq \ell_{2}$ we have

$$
x_{\ell} \leq \frac{x_{\ell-1}}{2}+c+\sum_{j=\ell_{1}}^{\ell-1} b_{j} x_{j} .
$$

Then $x_{\ell_{2}} \leq 2\left(x_{\ell_{1}}+c\right) \exp \left(3 \sum_{j=\ell_{1}}^{\ell_{2}-1} b_{j}\right)$.

Now we are ready to prove Proposition 19 .

Proof of Proposition 19 We will adapt the proof of Proposition 28 from [13]. Let $a=\alpha_{\ell_{1}, \lambda}$ and define $a_{\diamond}, a^{\diamond} \in 2 \pi \mathbb{Z}$ so that $\left[a_{\diamond}, a^{\diamond}\right)$ is an interval of length $2 \pi$ containing $a$. We can assume that $\lambda \geq 0$, the other case being very similar. We will drop the index $\lambda$ from $\alpha$ and we will write $\mathbb{E}()=.\mathbb{E}\left(. \mid \mathscr{F}_{\ell_{1}}\right)$.

We will show that there exists $c_{0}$ so that if $\mathscr{K}>c_{0}$, then if $\tilde{a}=a_{\diamond}$ or $a^{\diamond}$ then

$$
\mathbb{E}\left|\alpha_{\ell_{2}}-\tilde{a}\right| \leq c_{1}(|a-\tilde{a}|+\sqrt{\varepsilon}+y) .
$$

The claim of the proposition follows from this by an application of the triangle inequality, the additional condition $\kappa>c_{0}$ is treated via the error term $1 / \kappa$. 
In order to prove (74) for $\tilde{a}=a_{\diamond}$ we follow the steps described in Proposition 28 from [13]. Using the exact same argument we only need to prove that for the coefficients $b_{\ell}$ in Lemma 21 are bounded by a constant depending only on $\bar{\lambda}, \beta$ and that $\alpha$ never goes below an integer multiple of $2 \pi$ that it passes.

The first statement is easy to check, we have,

$$
\sum_{\ell=\ell_{1}}^{\ell_{2}-1} b_{\ell} \leq c_{0}\left(n_{1}^{1 / 2}\left(n_{1}^{-1 / 2} \wedge 1\right)+\left(n_{1}^{-1 / 6} \wedge 1\right)+\left(n_{1}^{1 / 3} \vee 1\right)\left(n_{1}^{-1 / 3} \wedge 1\right)+\#(J)\right)<c^{\prime} .
$$

To prove the other statement first recall the evolution steps (63) and that $\alpha_{j, \lambda}=\varphi_{j, \lambda}-\varphi_{j, \lambda}$ for $j \in \mathbb{Z} / 4$. Using the fact that the maps $\mathbf{L}_{\ell, \lambda}, \hat{\mathbf{L}}_{\ell, \lambda}$ and their conjugates are monotone in $\lambda$ (as functions

on $\mathbb{R}$ ) we get that $\alpha_{\ell, \lambda} \leq \alpha_{\ell+1 / 4, \lambda}$ and $\alpha_{\ell+1 / 2, \lambda} \leq \alpha_{\ell+3 / 4, \lambda}$. Moreover, since $\left(\mathbf{S}_{\ell, 0}\right)^{\hat{\mathbf{Q}}_{\ell}}$ and $\left(\hat{\mathbf{S}}_{\ell, 0}\right)^{\mathbf{Q}_{\ell}}$ are $2 \pi$-quasiperiodic functions on $\mathbb{R}$ we have $\left\lfloor\alpha_{\ell+1 / 4, \lambda}\right\rfloor_{2 \pi}=\left\lfloor\alpha_{\ell+1 / 2, \lambda}\right\rfloor_{2 \pi}$ and $\left\lfloor\alpha_{\ell+3 / 4, \lambda}\right\rfloor_{2 \pi}=\left\lfloor\alpha_{\ell+1, \lambda}\right\rfloor_{2 \pi}$. Hence, we get the following inequality:

$$
\left\lfloor\alpha_{\ell, \lambda}\right\rfloor_{2 \pi} \leq\left\lfloor\alpha_{\ell+1 / 4, \lambda}\right\rfloor_{2 \pi}=\left\lfloor\alpha_{\ell+1 / 2, \lambda}\right\rfloor_{2 \pi} \leq\left\lfloor\alpha_{\ell+3 / 4, \lambda}\right\rfloor_{2 \pi}=\left\lfloor\alpha_{\ell+1, \lambda}\right\rfloor_{2 \pi},
$$

which implies that $\alpha$ never goes below an integer multiple of $2 \pi$ that it passes. This means $\alpha_{\ell} \geq a_{\diamond}$ and $\alpha_{\ell}-a_{\diamond} \geq \hat{\alpha}_{\ell}$ for $\ell \geq \ell_{1}$.

Lemma 21 provides the bound

$$
\left|\mathbb{E} \alpha_{\ell}-a_{\diamond}\right| \leq\left(a-a_{\diamond}\right)+c(y+\sqrt{\varepsilon})+\mathbb{E} \hat{\alpha}_{\ell-1} / 2+\sum_{j=\ell_{1}}^{\ell-2} b_{j} \mathbb{E} \hat{\alpha}_{j} .
$$

Since $\left|\mathbb{E} \alpha_{\ell}-a_{\diamond}\right| \geq \mathbb{E} \hat{\alpha}_{\ell}$, inequality (74) follows for $\tilde{a}=a_{\diamond}$ via Lemma 22 .

In order to deal with the $\tilde{a}=a^{\diamond}$ case in (74) we define $T \in \mathbb{Z} / 2$ the first time when $\alpha_{T} \geq a^{\diamond}$. Note that $\alpha$ can only pass an integer multiple of $2 \pi$ in the $\ell \rightarrow \ell+1 / 4$ or $\ell+1 / 2 \rightarrow \ell+3 / 4$ steps, and $\varphi$ evolves deterministically in these steps. This means that $T-1 / 2$ is a stopping time with respect to $\mathscr{F}_{j}, j \in \mathbb{Z} / 2$.

For large enough $\mathscr{K}$ we have $\frac{\bar{\lambda}}{4 \sqrt{n_{0} k}} \leq \frac{1}{10}$. Then by Lemma 12 we get the uniform bound

$$
\alpha_{j+1 / 4, \lambda}-\alpha_{j, \lambda} \leq c n_{0}^{-1 / 2}, \quad j \leq \ell_{2}, j \in \mathbb{Z} / 2 .
$$

By the strong Markov property and the bound (60) we get

$$
\mathbb{E}\left[\left(\alpha_{T}-a^{\diamond}\right) \mathbf{1}\left(T \leq \ell_{2}\right)\right] \leq c n_{0}^{-1 / 2} \text { and } \mathbb{E}\left[\left(\alpha_{T+1 / 2}-a^{\diamond}\right) \mathbf{1}\left(T \leq \ell_{2}\right)\right] \leq c n_{0}^{-1 / 2} .
$$

Using this together with the first part of the proof and the strong Markov property again we get

$$
\begin{aligned}
\mathbb{E}\left(\alpha_{\ell_{2}}-a^{\diamond}\right)^{+} & =\mathbb{E}\left[\mathbf{1}\left(T \leq \ell_{2}\right) \mathbb{E}\left[\alpha_{\ell_{2}}-a^{\diamond} \mid \mathscr{F}_{T}\right]\right] \\
& \leq c_{1}\left(\mathbb{E}\left[\left(\alpha_{T}-a^{\diamond}\right) \mathbf{1}\left(T \leq \ell_{2}\right)\right]+\sqrt{\varepsilon}+y\right) \\
& \leq c_{1}^{\prime}(\sqrt{\varepsilon}+y),
\end{aligned}
$$

Lemma 21 gives

$$
\left|\mathbb{E} \alpha_{\ell}-a^{\diamond}\right| \leq\left(a^{\diamond}-a\right)+c(y+\sqrt{\varepsilon})+\mathbb{E} \hat{\alpha}_{\ell-1} / 2+\sum_{j=\ell_{1}}^{\ell-2} b_{j} \mathbb{E} \hat{\alpha}_{j}
$$


Then by (75) and the identity $|a|=-a+2 a^{+}$we get

$$
\begin{aligned}
\mathbb{E}\left|\alpha_{\ell}-a^{\diamond}\right| & \leq\left|\mathbb{E} \alpha_{\ell}-a^{\diamond}\right|+2 \mathbb{E}\left(\alpha_{\ell}-a^{\diamond}\right)^{+} \\
& \leq\left(a^{\diamond}-a\right)+c(y+\sqrt{\varepsilon})+\mathbb{E} \hat{\alpha}_{\ell-1} / 2+\sum_{j=\ell_{1}}^{\ell-2} b_{j} \mathbb{E} \hat{\alpha}_{j} .
\end{aligned}
$$

Since $\hat{\alpha}_{\ell} \leq\left|\alpha_{\ell}-a^{\diamond}\right|$, the Gronwall-type estimate in Lemma 22 implies (74) with $\tilde{a}=a^{\diamond}$.

\subsection{The uniform limit}

Proposition 23. Assume that $\mathscr{K}=\mathscr{K}(n) \rightarrow \infty$ and that $\mathscr{K} n_{0}^{-1}\left(n_{1}^{1 / 3} \vee 1\right) \rightarrow 0$ as $n \rightarrow \infty$. Then, $\varphi_{n_{2}, 0}$ modulo $2 \pi$ converges in distribution to the uniform distribution on $(0,2 \pi)$ as $n \rightarrow \infty$.

Proof. We can use exactly the same argument as in Proposition 33, [13]. We show that given $\epsilon>0$, every subsequence has a further subsequence along which $\varphi_{n_{2}, 0}$ modulo $2 \pi$ is eventually $\epsilon$-close to the uniform distribution. We set $\xi=\left\lfloor\mathscr{K}\left(n_{1}^{1 / 3} \vee 1\right)\right\rfloor$ and pick $\tau=\tau(\epsilon)$ with $\tau \xi \leq n_{2}$. Because of $\mathscr{K} n_{0}^{-1}\left(n_{1}^{1 / 3} \vee 1\right) \rightarrow 0$ we will be able to let $\tau \rightarrow \infty$. We will show that for any fixed $\tau$ the distribution of $\varphi_{n_{2}, 0}-\varphi_{n_{2}-\tau \xi, 0}$ given $\mathscr{F}_{n_{2}-\tau \xi}$ is asymptotically normal with a variance going to $\infty$ as $\tau \rightarrow \infty$. From that the statement will follow.

Note that the arguments of Proposition 17 can be repeated for the evolution of $\varphi_{n_{2}-\tau \xi+\ell, 0}$ with $0 \leq \ell \leq \tau \xi$ which gives that $\varphi_{n_{2}, 0}$ conditioned on $\mathscr{F}_{n_{2}-\tau \xi}$ converges to $\varphi_{0}\left(1-(1+\tau)^{-1}\right)$ where $\varphi_{0}(t)$ is the solution of (57) with $\lambda=0$. This is just a normal random variable, its variance is given by the integrating the sum of the squares of the independent diffusion coefficients on $\left[0,1-(1+\tau)^{-1}\right]$. This is at least as big as the variance coming from the $d W$ term which gives $\int_{0}^{1-(1+\tau)^{-1}} \frac{2}{\beta(1-t)} d t$. This goes to $\infty$ if $\tau \rightarrow \infty$ as required.

\section{Last stretch}

The purpose of this section is to prove that on the interval $\left[n_{2}, n\right]$ the relative target phase function $\alpha_{\ell, \lambda}^{\odot}=\varphi_{\ell, \lambda}^{\odot}-\varphi_{\ell, 0}^{\odot}$ does not change much.

Proposition 24. For any fixed $\lambda \in \mathbb{R}$ and $\mathscr{K}>0$ we have $\alpha_{n_{2}, \lambda}^{\odot} \stackrel{P}{\longrightarrow} 0$ as $n \rightarrow \infty$.

The length of the interval $\left[n_{2}, n\right]$ is equal to $n_{1}+\mathscr{K}\left(n_{1}^{1 / 3} \vee 1\right)$, up to an error of order 1 . By taking an appropriate subsequence of $n$ (see Remark 10) we may assume that $n_{1}$ has a finite or infinite limit as $n \rightarrow \infty$. We will consider these two cases separately.

Proof of Proposition 24 in the $\lim _{n \rightarrow \infty} n_{1}<\infty$ case. By (12) we have that $\left|m-n-\mu_{n}^{2}\right| / \mu_{n}$ converges as $n \rightarrow \infty$, by taking an appropriate subsequence we can assume that the limit also exists without the absolute values. Note that condition (10) implies that $\lim _{n \rightarrow \infty} m / n>1$ and thus $m-n \rightarrow \infty$.

We may also assume that $n-n_{2}$ is eventually equal to an integer $\xi$. From there we proceed similarly as in [13]. We first note that by (36) and (39) we have

$$
\varphi_{n-\xi, \lambda}^{\odot} \mathbf{Q}_{n-\xi-1}^{-1}=0 * \mathbf{R}_{n-1, \lambda} \ldots \mathbf{R}_{n-\xi, \lambda} \mathbf{T}_{n-\xi}
$$


where $\mathbf{R}_{\ell, \lambda}=\hat{\mathbf{M}}_{\ell}^{-1} \hat{\mathbf{J}}_{\ell}^{-1} \mathbf{M}_{\ell}^{-1} \mathbf{J}_{\ell}^{-1}$. From (31) and 32 ) we get

$$
r . \mathbf{R}_{n-j, \lambda}=\frac{r s_{n-j}^{2}+r s_{n-j} Y_{n-j}-s_{n-j} \Lambda}{p_{n-j} p_{n-j+1}-\Lambda^{2}+p_{n-j+1} X_{n-j}+r s_{n-j} \Lambda+r Y_{n-j} \Lambda}
$$

where $\Lambda=\mu_{n}+\lambda /\left(4 n_{0}^{1 / 2}\right)$. Using $(12), s_{n-j}=\sqrt{j-1 / 2}, p_{n-j}=\sqrt{m-n+j-1 / 2}$ and $m-n \rightarrow \infty$ we get that $r . \mathbf{R}_{n-j, \lambda}-r \cdot \mathbf{R}_{n-j, 0} \stackrel{P}{\longrightarrow} 0$ for any fixed $j$. This leads to

$$
\alpha_{n_{2}, \lambda}^{\odot}=\varphi_{n-\xi, \lambda}^{\odot} * \mathbf{Q}_{n-\xi-1}^{-1}-\varphi_{n-\xi, 0}^{\odot} * \mathbf{Q}_{n-\xi-1}^{-1} \rightarrow 0 \text { as } n \rightarrow \infty
$$

If $\lim _{n \rightarrow} n_{1}=\infty$ then we will need the edge scaling results proved in [9] which are summarized in Theorem 3 of the introduction. The initial condition $f(0)=0, f^{\prime}(0)=1$ for the operator $\mathscr{H}_{\beta}$ in the theorem comes from the fact that the discrete eigenvalue equation for $A_{n, m} A_{n, m}^{T}$ with an eigenvalue $\Lambda^{\prime}=(\sqrt{n}+\sqrt{m})^{2}+\frac{(\sqrt{m}+\sqrt{n})^{4 / 3}}{(m n)^{1 / 6}} v$ is equivalent to a three-term recursion for the vector entries $w_{\ell, \Lambda}$ (c.f. (18)) with the initial condition $w_{0, v}=0$ and $w_{1, v} \neq 0$.

By [9], Remark 3.8, the results of [9] extend to solutions of the same three-term recursion with more general initial conditions. We say that a value of $v$ is an eigenvalue for a family of recursions parametrized by $v$ if the corresponding recursion reaches 0 in its last step. Suppose that for given $\zeta \in[-\infty, \infty]$ the initial condition for the three-term recursion equation satisfies

$$
\frac{(m n)^{-1 / 3}}{(\sqrt{m}+\sqrt{n})^{-2 / 3}} \frac{w_{0, v}}{\left(w_{1, v}-w_{0, v}\right)}=\frac{(m n)^{-1 / 3}}{(\sqrt{m}+\sqrt{n})^{-2 / 3}}\left(r_{0, v}-1\right)^{-1} \stackrel{P}{\longrightarrow} \zeta,
$$

uniformly in $v$ with $r_{0}:=w_{1, v} / w_{0, v}$. Here the factor $\frac{(m n)^{1 / 3}}{(\sqrt{m}+\sqrt{n})^{2 / 3}}$ is the spatial scaling for the problem ([9], Section 5). Then the eigenvalues of this family of recursions converge to those of the stochastic Airy operator with initial condition $f(0) / f^{\prime}(0)=\zeta$. The corresponding point process $\Xi_{\zeta}$ is also a.s. simple (i.e. there are no multiple points) and it will satisfy the following non-atomic property: for any $x \in \mathbb{R}$ we have $\mathrm{P}\left(x \in \Xi_{\zeta}\right)=0$ (see [9], Remark 3.8). Similar statement holds at the lower edge if $\liminf _{n \rightarrow \infty} m / n>1$ with $\left(r_{n, v}+1\right)^{-1}$ in $(76)$. (In this case one first multiplies the off-diagonal entries of $A_{n, m} A_{n, m}^{T}$ by -1 before applying the arguments of [9], this will not change the eigenvalues.)

If $m / n \rightarrow \gamma \in[1, \infty)$ then we can rewrite (76) jointly for the upper and lower soft edge as

$$
\frac{\gamma^{-1 / 3}}{(\sqrt{\gamma} \pm 1)^{-2 / 3}} n^{-1 / 3}\left(r_{0, v} \mp 1\right)^{-1} \stackrel{P}{\longrightarrow} \zeta,
$$

The multiplier is 1 in the case $\gamma=\infty$ and it is always a finite nonzero value unless $\gamma=1$ and we are at the lower edge.

Proof of Proposition 24 if $\lim _{n \rightarrow \infty} n_{1}=\infty$. By taking an appropriate subsequence, we may assume that $\mu_{n}-\sqrt{m-n}$ is always positive or always negative. According to the proof of Lemma 34 in [13] we need to consider the family of recursions

$$
r_{\ell+1, v}=r_{\ell, v} . \mathbf{J}_{\ell} \mathbf{M}_{\ell} \hat{\mathbf{J}}_{\ell} \hat{\mathbf{M}}_{\ell}, \quad n_{2} \leq \ell \leq n
$$


with initial condition

$$
r_{n_{2}, v}=x \cdot \mathbf{T}_{n_{2}}^{-1}=\mathfrak{I}\left(\rho_{n_{2}}\right) x+\Re\left(\rho_{n_{2}}\right)
$$

for a given $x \in \mathbb{R}$ and show that the probability of having an eigenvalue in $[0, \lambda]$ converges to 0 as $n \rightarrow \infty$.

We introduce

$$
n^{*}=n-n_{2}, \quad m^{*}=m-n_{2} .
$$

Note that the recursion is determined by the bottom $\left(2 n^{*}\right) \times\left(2 n^{*}\right)$ submatrix of $\tilde{A}_{n, m}^{D^{(n)}}$ in 29 , which has the exact same distribution as the matrix $\tilde{A}_{n^{*}, m^{*}}^{\left.D^{*}\right)}$. Thus we can consider the eigenvalue equation for $\tilde{A}_{n^{*}, m^{*}}^{D^{\left(n^{*}\right)}}$ with generalized initial condition

$$
r_{0, \lambda}^{*}=\mathfrak{I}\left(\rho_{n_{2}}\right) x+\mathfrak{R}\left(\rho_{n_{2}}\right) .
$$

(We will use $*$ to denote that we are working with the smaller matrix.) We will transform this back to an eigenvalue equation for $\tilde{A}_{n^{*}, m^{*}}$ and then $A_{n^{*}, m^{*}} A_{n^{*}, m^{*}}^{T}$ with a generalized initial condition.

Recall the recursion (31) and that $r_{\ell}^{*}=u_{\ell+1}^{*} / v_{\ell}^{*}, \hat{r}_{\ell}^{*}=v_{\ell}^{*} / u_{\ell}^{*}$. From this we get

$$
\begin{aligned}
\frac{v_{1}^{*}}{v_{0}^{*}} & =r_{0}^{*} \hat{r}_{1}^{*}=r_{0}^{*}\left(-\frac{1}{r_{0}^{*}} \cdot \frac{s_{0}^{*}}{p_{0}^{*}}+\frac{\Lambda}{p_{0}^{*}}\right)\left(1+\frac{X_{0}^{*}}{p_{0}^{*}}\right)^{-1} \\
& =\left(-\frac{s_{0}^{*}}{p_{0}^{*}}+\left(\mathfrak{S}\left(\rho_{n_{2}}\right) x+\Re\left(\rho_{n_{2}}\right)\right) \frac{\Lambda}{p_{0}^{*}}\right) \frac{\beta p_{1}^{*} p_{0}^{*}}{\tilde{\chi}_{\beta\left(m^{*}-1\right)}^{2}}
\end{aligned}
$$

where $\Lambda=\mu_{n}+\frac{\lambda}{4 \sqrt{n_{0}}}$. Denote the solution of the generalized eigenvalue equation for $\tilde{A}_{n^{*}, m^{*}}$ corresponding to $\Lambda$ with $\tilde{u}_{1}^{*}, \tilde{v}_{1}^{*}, \ldots$ If we remove the conjugation with $D^{\left(n^{*}\right)}$ from $\tilde{A}_{n^{*}, m^{*}}^{\left.D^{*}\right)}$ then from 78 , we get

$$
\frac{\tilde{v}_{1}^{*}}{\tilde{v}_{0}^{*}}=\left(-\frac{s_{0}^{*}}{p_{0}^{*}}+\left(\mathfrak{I}\left(\rho_{n_{2}}\right) x+\Re\left(\rho_{n_{2}}\right)\right) \frac{\Lambda}{p_{0}^{*}}\right) \frac{\chi_{\beta\left(n^{*}-1\right)} p_{0}^{*}}{\tilde{\chi}_{\beta\left(m^{*}-1\right)} s_{1}^{*}} .
$$

By Remark 8 this is exactly the initial condition for the generalized eigenvalue equation for $A_{n^{*}, m^{*}} A_{n^{*}, m^{*}}^{T}$ with eigenvalue $\Lambda^{2}=\mu_{n}^{2}+\frac{\mu_{n} \lambda}{2 \sqrt{n_{0}}}+\frac{\lambda^{2}}{16 n_{0}}$.

The spectrum of the matrix $A_{n^{*}, m^{*}} A_{n^{*}, m^{*}}^{T}$ is concentrated asymptotically to $\left[\left(\sqrt{m^{*}}-\sqrt{n^{*}}\right)^{2},\left(\sqrt{m^{*}}+\right.\right.$ $\left.\left.\sqrt{n^{*}}\right)^{2}\right]$. A direct computation shows that $\sqrt{m_{1}} \pm \sqrt{n_{1}}=\mu_{n}$, where we have + if $\mu_{n}-\sqrt{m-n}>0$ and - otherwise. This means that if $\mu_{n}<\sqrt{m-n}$ then our original bulk scaling around $\mu_{n}$ corresponds to the lower edge scaling of $A_{n^{*}, m^{*}} A_{n^{*}, m^{*}}^{T}$ and if $\mu_{n}>\sqrt{m-n}$ then we get the upper edge scaling. (Note that because of our assumptions if $\mu_{n}<\sqrt{m-n}$ than $\liminf m / n>1$.)

Again, by taking an appropriate subsequence, we may assume that $m^{*} / n^{*} \rightarrow \gamma^{*} \in[1, \infty]$. We first check that the initial condition 79 satisfies $(77)$, this is equivalent to showing that $\left(n^{*}\right)^{1 / 3}\left(\frac{\tilde{v}_{1}^{*}}{\tilde{v}_{0}^{*}} \mp 1\right)$ converges in probability to a constant. Since $n_{1} \rightarrow \infty$ we have

$$
\begin{aligned}
& m^{*}> n^{*} \rightarrow \infty, \quad \rho_{n_{2}}= \pm 1+i \sqrt{\mathscr{K}}\left(n^{*}\right)^{-1 / 3}+o\left(\left(n^{*}\right)^{-1 / 3}\right), \\
& n_{1}=n^{*}-\mathscr{K}\left(n^{*}\right)^{1 / 3}, \quad m_{1}=m^{*}-\mathscr{K}\left(n^{*}\right)^{1 / 3}, \\
& \mu_{n}=\sqrt{m^{*}} \pm \sqrt{n^{*}}-\frac{\mathscr{K}}{2}\left(n^{*}\right)^{1 / 3}\left(\left(m^{*}\right)^{-1 / 2} \pm\left(n^{*}\right)^{-1 / 2}\right)+o\left(\left(n^{*}\right)^{-2 / 3}\right) .
\end{aligned}
$$


Since $\ell^{-1 / 2} \chi_{\ell} \stackrel{P}{\longrightarrow} 1$ as $\ell \rightarrow \infty$ we get that in probability

$$
\lim \left(n^{*}\right)^{1 / 3}\left(\frac{\tilde{v}_{1}^{*}}{\tilde{v}_{0}^{*}} \mp 1\right)=\sqrt{\mathscr{K}} x\left(1 \pm\left(\gamma^{*}\right)^{-1 / 2}\right)
$$

and the convergence is uniform if we assume that $\lambda$ is bounded. This means that we may apply the edge scaling result, we just need to convert the scaling from the bulk to the edge. In the bulk we were interested in the interval $I=\left[\mu_{n}^{2},\left(\mu_{n}+\lambda n_{0}^{-1 / 2} / 4\right)^{2}\right]$. If we apply the edge scaling, then this interval becomes $\frac{\left(m^{*} n^{*}\right)^{1 / 6}}{\left(\sqrt{\left.m^{*} \pm \sqrt{n^{*}}\right)^{4 / 3}}\right.}\left(I-\left(\sqrt{m^{*}} \pm \sqrt{n^{*}}\right)^{2}\right)$. Using our asymptotics for $\mu_{n}$ we get that for the end point of the interval

$$
\frac{\left(m^{*} n^{*}\right)^{1 / 6}}{\left(\sqrt{m^{*}} \pm \sqrt{n^{*}}\right)^{4 / 3}}\left(\mu_{n}^{2}-\left(\sqrt{m^{*}} \pm \sqrt{n^{*}}\right)^{2}\right) \rightarrow-\frac{\mathscr{K}}{2}\left(1 \pm\left(\gamma^{*}\right)^{-1 / 2}\right)^{1 / 6}
$$

and for the length we get

$$
\lim _{n \rightarrow \infty}\left(\left(\mu_{n}+\lambda n_{0}^{-1 / 2} / 4\right)^{2}-\mu_{n}^{2}\right) \frac{\left(m^{*} n^{*}\right)^{1 / 6}}{\left(\sqrt{m^{*}} \pm \sqrt{n^{*}}\right)^{4 / 3}}=\frac{\left(\gamma^{*}\right)^{1 / 6}}{\left(\sqrt{\gamma^{*}} \pm 1\right)^{1 / 3}} \lim _{n \rightarrow \infty} \frac{\lambda}{\sqrt{n_{0}}}=0 .
$$

(We used that if $\gamma^{*}=1$ then we must have + in the \pm .) This means that after the edge rescaling the interval shrinks to a point, meaning that the probability that our original recursion has an eigenvalue in $[0, \lambda]$ converges to the probability that the limiting edge point process has a point at a given value which is equal to 0 .

\section{Appendix}

In this section we provide the needed oscillation estimates.

Lemma 25. Suppose that $2 \pi>\theta_{1}>\theta_{2}>\ldots>\theta_{m}>0$ and let $s_{\ell}=\sum_{j=1}^{\ell} \theta_{j}$. Then

$$
\left|\sum_{j=1}^{m} e^{i s_{j}}\right| \leq c\left(\theta_{m}^{-1}+\left(2 \pi-\theta_{1}\right)^{-1}\right) \leq c^{\prime}\left(\left|e^{i \theta_{m} / 2}-1\right|^{-1}+\left|e^{i \theta_{1} / 2}+1\right|^{-1}\right) .
$$

Proof. The first inequality is the same as Lemma 36 from [13] and the second inequality is straightforward.

Lemma 26. Let $1 \leq \ell_{1}<\ell_{2} \leq n_{0}$ and $F_{\ell_{1}, \ell_{2}}^{(i)}=\sum_{j=\ell_{1}}^{\ell_{2}} \eta_{j}^{i}$ for $i=1,2$ and $g_{\ell} \in \mathbb{C}$. Then for $i=1,2$ :

$$
\begin{aligned}
& \left|\Re \sum_{\ell=\ell_{1}}^{\ell_{2}} g_{\ell} \eta_{\ell}^{i}\right| \leq\left|F_{\ell_{1}, \ell_{2}}^{(i)}\right|\left|g_{\ell_{2}}\right|+\sum_{\ell=\ell_{1}}^{\ell_{2}-1}\left|F_{\ell_{1}, \ell}^{(i)}\right|\left|g_{\ell+1}-g_{\ell}\right|, \\
& \left|\Re \sum_{\ell=\ell_{1}}^{\ell_{2}} g_{\ell} \eta_{\ell}^{i}\right| \leq\left|F_{\ell_{1}, \ell_{2}}^{(i)}\right| g_{\ell_{1}}||+\sum_{\ell=\ell_{1}}^{\ell_{2}-1}\left|F_{\ell+1, \ell_{2}}^{(i)}\right|\left|g_{\ell+1}-g_{\ell}\right| .
\end{aligned}
$$


We also have the following estimates:

$$
\begin{gathered}
\left|F_{\ell_{1}, \ell_{2}}^{(1)}\right| \leq c\left(1+n_{1}^{1 / 2} k_{2}^{-1 / 2}\right) \\
\left|F_{\ell_{1}, \ell_{2}}^{(2)}\right| \leq \begin{cases}c\left(\left|\rho_{\ell_{1}}^{2} \hat{\rho}_{\ell_{1}}^{2}+1\right|^{-1}+1+n_{1}^{1 / 2} k_{2}^{-1 / 2}\right) & \text { if }(\star) \text { or }(\star \star), \\
c\left(\left|\rho_{\ell_{2}}^{2} \hat{\rho}_{\ell_{2}}^{2}+1\right|^{-1}+1+n_{1}^{1 / 2} k_{1}^{-1 / 2}\right) & \text { if }(\star \star \star)\end{cases}
\end{gathered}
$$

where the conditions are given by

$$
\begin{aligned}
(\star): & \mu_{n} \geq \sqrt{m-n} \text { with } k_{0}, k_{1} \geq \sqrt{m_{1} n_{1}} \text { or } k_{0}, k_{1} \leq \sqrt{m_{1} n_{1}}, \\
(\star \star) & : \mu_{n}<\sqrt{m-n} \text { with } k_{0}, k_{1} \geq \sqrt{m_{1} n_{1}} \\
(\star \star \star) & : \mu_{n}<\sqrt{m-n} \text { with } k_{0}, k_{1} \leq \sqrt{m_{1} n_{1}} .
\end{aligned}
$$

Proof. The bounds (80) and (81) follow from partial summation. In order to prove the bounds on $F_{\ell_{1}, \ell_{2}}^{(i)}$ we will apply Lemma 25 , but we need to consider various cases. Note that the constant $c$ might change from line to line.

Case 1: $\mu_{n} \geq \sqrt{m-n}, \Re \rho_{\ell} \geq 0$.

We have the bounds

$$
\begin{array}{cc}
k^{1 / 2}\left(n_{1}+k\right)^{-1 / 2} \leq \arg \rho_{\ell}, & n_{1}^{1 / 2}\left(n_{1}+k\right)^{-1 / 2} \leq \pi / 2-\arg \rho_{\ell}, \\
k^{1 / 2}\left(m_{1}+k\right)^{-1 / 2} \leq \arg \hat{\rho}_{\ell}, & m_{1}^{1 / 2}\left(m_{1}+k\right)^{-1 / 2} \leq \pi / 2-\arg \hat{\rho}_{\ell} .
\end{array}
$$

and $\arg \left(\rho_{\ell} \hat{\rho}_{\ell}\right)$ is decreasing. The sequence $\arg \left(\hat{\rho}_{\ell}^{2} \rho_{\ell}^{2}\right)$ satisfies the conditions of Lemma 25 and using $m_{1}>n_{1}$ and $m_{1}>c n>c n_{0}$ we get the bound

$$
\left|\sum_{\ell=\ell_{1}}^{\ell_{2}} \eta_{\ell}\right| \leq c\left(\left(n_{1}+k_{2}\right)^{1 / 2} k_{2}^{-1 / 2}+\left(k_{1}+m_{1}\right)^{1 / 2} m_{1}^{-1 / 2}\right) \leq c\left(n_{1}^{1 / 2} k_{2}^{-1 / 2}+1\right) .
$$

We have

$$
\rho_{\ell} \hat{\rho}_{\ell}=\frac{\sqrt{m_{1} n_{1}}-k}{\sqrt{k+m_{1}} \sqrt{k+n_{1}}}+\frac{i \sqrt{k}\left(\sqrt{m_{1}}+\sqrt{n_{1}}\right)}{\sqrt{k+m_{1}} \sqrt{k+n_{1}}}
$$

which means that if $k_{1}, k_{2} \geq \sqrt{m_{1} n_{1}}$ or $k_{1}, k_{2} \leq \sqrt{m_{1} n_{1}}$ then we can use Lemma 25 for the sequence $\arg \left(\hat{\rho}_{\ell}^{4} \rho_{\ell}^{4}\right)$. (In the first case $\pi / 2 \leq \arg \left(\hat{\rho}_{\ell} \rho_{\ell}\right)<\pi$ while in the second case $0<\arg \left(\hat{\rho}_{\ell} \rho_{\ell}\right) \leq \pi / 2$.) From the lemma we get

$$
\left|\sum_{\ell=\ell_{1}}^{\ell_{2}} \eta_{\ell}^{2}\right| \leq c\left(\left|\rho_{\ell_{1}}^{2} \hat{\rho}_{\ell_{1}}^{2}+1\right|^{-1}+\left|\rho_{\ell_{2}}^{2} \hat{\rho}_{\ell_{2}}^{2}-1\right|^{-1}\right)
$$

and explicit computation together with $m_{1}>c n>c k$ gives

$$
\left|\rho_{\ell}^{2} \hat{\rho}_{\ell}^{2}-1\right|^{-1}=\frac{\sqrt{k+m_{1}} \sqrt{k+n_{1}}}{2 \sqrt{k}\left(\sqrt{m_{1}}+\sqrt{n_{1}}\right)} \leq c\left(1+n_{1}^{1 / 2} k^{-1 / 2}\right) .
$$


This finishes the proof of the lemma in this case.

Case 2: $\mu_{n}<\sqrt{m-n}, \Re \rho_{\ell}<0$.

Now we have

$$
\rho_{\ell} \hat{\rho}_{\ell}=-\frac{k+\sqrt{m_{1}} \sqrt{n_{1}}}{\sqrt{k+m_{1}} \sqrt{k+n_{1}}}+\frac{i \sqrt{k}\left(\sqrt{m_{1}}-\sqrt{n_{1}}\right)}{\sqrt{k+m_{1}} \sqrt{k+n_{1}}} .
$$

meaning $\pi / 2<\arg \left(\hat{\rho}_{\ell} \rho_{\ell}\right)<\pi$. Differentiation of the real part shows that $\arg \rho_{\ell} \hat{\rho}_{\ell}$ decreases if $k>$ $\sqrt{m_{1} n_{1}}$ and then it increases. This means that we can apply Lemma 25 for the sequences $\arg \left(\hat{\rho}_{\ell}^{2} \rho_{\ell}^{2}\right)$ or $\arg \left(\hat{\rho}_{\ell}^{4} \rho_{\ell}^{4}\right)$ if $k_{1}, k_{2} \geq \sqrt{n_{1} m_{1}}$ and the reversed versions of these sequences if $k_{1}, k_{2} \leq \sqrt{n_{1} m_{1}}$. From (85) we get

$$
\pi \leq \arg \rho_{\ell}^{2} \hat{\rho}_{\ell}^{2}, \quad 2 \pi-\arg \rho_{\ell}^{2} \hat{\rho}_{\ell}^{2} \geq \frac{2 \sqrt{k}\left(\sqrt{m_{1}}-\sqrt{n_{1}}\right)}{\sqrt{k+m_{1}} \sqrt{k+n_{1}}} \geq c\left(k^{-1 / 2} n_{1}^{1 / 2}+1\right)^{-1}
$$

where we used $\sqrt{m_{1}-n_{1}} \geq c \sqrt{m_{1}}$ which follows from $m_{1}>c n>c n_{1}$. Applying Lemma 25 to $\arg \left(\hat{\rho}_{\ell}^{2} \rho_{\ell}^{2}\right)$ (or the reversed sequence) we get

$$
\left|\sum_{\ell=\ell_{1}}^{\ell_{2}} \eta_{\ell}\right| \leq \begin{cases}c\left(n_{1}^{1 / 2} k_{1}^{-1 / 2}+1\right) & \text { if } k_{1}, k_{2} \geq \sqrt{n_{1} m_{1}} \\ c\left(n_{1}^{1 / 2} k_{2}^{-1 / 2}+1\right) & \text { if } k_{1}, k_{2} \leq \sqrt{n_{1} m_{1}}\end{cases}
$$

Noting that $k_{1}^{-1 / 2} \leq k_{2}^{-1 / 2}$ this proves $(82)$ in Case 2 if $k_{1}, k_{2} \leq \sqrt{n_{1} m_{1}}$ or $k_{1}, k_{2} \geq \sqrt{n_{1} m_{1}}$. If $k_{1}>$ $\sqrt{n_{1} m_{1}}$ and $k_{2}<\sqrt{n_{1} m_{1}}$ then we can cut the sum in to parts at $\sqrt{n_{1} m_{1}}$ and since for $k \geq \sqrt{m_{1} n_{1}}$ we have $n_{1}^{1 / 2} k^{-1 / 2} \leq 1$ we have (82) in this case as well.

To prove 83 we apply Lemma 25 to $\arg \left(\hat{\rho}_{\ell}^{4} \rho_{\ell}^{4}\right)$ (or its reversed) to get

$$
\left|\sum_{\ell=\ell_{1}}^{\ell_{2}} \eta_{\ell}^{2}\right| \leq \begin{cases}c\left(\left|\rho_{\ell_{1}}^{2} \hat{\rho}_{\ell_{1}}^{2}+1\right|^{-1}+\left|\rho_{\ell_{2}}^{2} \hat{\rho}_{\ell_{2}}^{2}-1\right|^{-1}\right) & \text { if } k_{1}, k_{2} \geq \sqrt{n_{1} m_{1}} \\ c\left(\left|\rho_{\ell_{2}}^{2} \hat{\rho}_{\ell_{2}}^{2}+1\right|^{-1}+\left|\rho_{\ell_{1}}^{2} \hat{\rho}_{\ell_{1}}^{2}-1\right|^{-1}\right) & \text { if } k_{1}, k_{2} \leq \sqrt{n_{1} m_{1}}\end{cases}
$$

From this (83) follows by noting that

$$
\left|\rho_{\ell}^{2} \hat{\rho}_{\ell}^{2}-1\right|^{-1}=\frac{\sqrt{k+m_{1}} \sqrt{k+n_{1}}}{2 \sqrt{k}\left(\sqrt{m_{1}}-\sqrt{n_{1}}\right)} \leq c\left(n_{1}^{1 / 2} k^{-1 / 2}+1\right) .
$$

where the first equality is explicit computation and the inequality is from $(86)$.

Acknowledgments. The authors wish to thank the anonymous referees for valuable comments and suggestions.

\section{References}

[1] Ioana Dumitriu and Alan Edelman. Matrix models for beta ensembles. J. Math. Phys., 43(11):5830-5847, 2002. MR1936554 
[2] Ioana Dumitriu and Peter Forrester. Tridiagonal realization of the anti-symmetric Gaussian $\beta$-ensemble J. Math. Phys., 51, 093302, 2010

[3] László Erdős, Benjamin Schlein, Horng-Tzer Yau, and Jun Yin. The local relaxation flow approach to universality of the local statistics for random matrices, 2009. Preprint, arXiv:0911.3687.

[4] Stewart N. Ethier and Thomas G. Kurtz. Markov processes. John Wiley \& Sons Inc., New York, 1986. MR0838085

[5] Peter Forrester. Log-gases and Random matrices. Princeton University Press, 2010. MR2641363

[6] Eugene Kritchevski, Benedek Valkó, and Bálint Virág. The scaling limit of the critical onedimensional random Schrödinger operator, 2010. Preprint.

[7] V. A. Marčenko and L. A. Pastur. Distribution of eigenvalues in certain sets of random matrices. Mat. Sb. (N.S.), 72 (114):507-536, 1967. MR0208649

[8] Madan Lal Mehta. Random matrices. Elsevier/Academic Press, 2004. MR2129906

[9] Jose A. Ramírez, Brian Rider, and Bálint Virág. Beta ensembles, stochastic Airy spectrum, and a diffusion, 2006. J. Amer. Math. Soc., To appear, arXiv:math.PR/0607331.

[10] Jose A. Ramírez and Brian Rider. Diffusion at the random matrix hard edge. Comm. Math. Phys., (288):887-906, 2009. MR2504858

[11] Daniel W. Stroock and S. R. Srinivasa Varadhan. Multidimensional diffusion processes. Classics in Mathematics. Springer-Verlag, Berlin, 1979. MR0532498

[12] Terence Tao and Van Vu. Random covariance matrices: Universality of local statistics of eigenvalues, 2009. Preprint: arXiv:0912.0966.

[13] Benedek Valkó and Bálint Virág. Continuum limits of random matrices and the Brownian carousel. Invent. Math., 177(3):463-508, 2009. MR2534097

[14] Benedek Valkó and Bálint Virág. Random Schrödinger operators on long boxes, noise explosion and the GOE, 2009. Preprint, arXiv:0912.0097.

[15] John Wishart. The generalized product moment distribution in samples from a normal multivariate population. Biometrika A, 20A:32-52, 1928. 